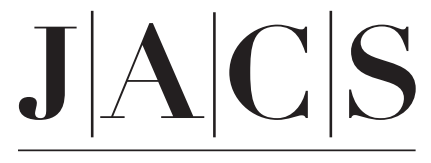

A R T I C L E S

Published on Web 09/25/2003

\title{
L-edge X-ray Absorption Spectroscopy of Non-Heme Iron Sites: Experimental Determination of Differential Orbital Covalency
}

\author{
Erik C. Wasinger, ${ }^{\dagger}$ Frank M. F. de Groot, ${ }^{\ddagger}$ Britt Hedman, ${ }^{*},+, \S$ \\ Keith O. Hodgson, ${ }^{*},+$, and Edward I. Solomon ${ }^{*},+$ \\ Contribution from the Department of Chemistry and Stanford Synchrotron Radiation Laboratory, \\ Stanford University, Stanford, California 94305 and Department of Inorganic Chemistry and \\ Catalysis, Utrecht University, Utrecht, The Netherlands \\ Received February 12, 2003; E-mail: Edward.Solomon@stanford.edu
}

\begin{abstract}
X-ray absorption spectroscopy has been utilized to obtain the L-edge multiplet spectra for a series of non-heme ferric and ferrous complexes. Using these data, a methodology for determining the total covalency and the differential orbital covalency (DOC), that is, differences in covalency in the different symmetry sets of the d orbitals, has been developed. The integrated L-edge intensity is proportional to the number of one-electron transition pathways to the unoccupied molecular orbitals as well as to the covalency of the iron site, which reduces the total L-edge intensity and redistributes intensity, producing shake-up satellites. Furthermore, differential orbital covalency leads to differences in intensity for the different symmetry sets of orbitals and, thus, further modifies the experimental spectra. The ligand field multiplet model commonly used to simulate L-edge spectra does not adequately reproduce the spectral features, especially the charge transfer satellites. The inclusion of charge transfer states with differences in covalency gives excellent fits to the data and experimental estimates of the different contributions of charge transfer shakeup pathways to the $t_{2 g}$ and $e_{g}$ symmetry orbitals. The resulting experimentally determined DOC is compared to values calculated from density functional theory and used to understand chemical trends in high- and low-spin ferrous and ferric complexes with different covalent environments. The utility of this method toward problems in bioinorganic chemistry is discussed.
\end{abstract}

\section{Introduction}

X-ray absorption techniques have been used extensively to develop electronic and geometric descriptions of metal sites. ${ }^{1,2}$ Specifically, extended X-ray absorption fine structure (EXAFS) at the iron K-edge has been an ideal tool for the determination of the local coordination environment at the iron site of a wide range of metalloproteins, ${ }^{3-7}$ model complexes, ${ }^{8,9}$ minerals,${ }^{10}$ and other materials. ${ }^{11}$ Additional electronic information has been

$\dagger$ Department of Chemistry, Stanford University.

$\div$ Utrecht University.

$\S$ Stanford Synchrotron Radiation Laboratory, Stanford University.

(1) Koningsberger, D. C., Prins, R., Eds. X-ray Absorption: Principles, Applications, Techniques of EXAFS, SEXAFS, and XANES; John Wiley \& Sons: New York, 1988; Vol. 92.

(2) Zhang, H. H.; Hedman, B.; Hodgson, K. O. In Inorganic Electronic Structure and Spectroscopy; Solomon, E. I., Lever, A. B. P., Eds.; John Wiley \& Sons: New York, 1999; pp 513-554.

(3) Loeb, K. E.; Westre, T. E.; Kappock, T. J.; Mitic, N.; Glasfeld, E. Caradonna, J. P.; Hedman, B.; Hodgson, K. O.; Solomon, E. I. J. Am. Chem Soc. 1997, 119, 1901-1915.

(4) Westre, T. E.; Loeb, K. E.; Zaleski, J. M.; Hedman, B.; Hodgson, K. O.; Solomon, E. I. J. Am. Chem. Soc. 1995, 117, 1309-1313.

(5) Wasinger, E.; Mitić, N.; Hedman, B.; Caradonna, J.; Solomon, E. I.; Hodgson, K. O. Biochemistry 2002, 41, 6211-6217.

(6) Davis, M. I.; Wasinger, E. C.; Westre, T. E.; Zaleski, J. M.; Orville, A M.; Lipscomb, J. D.; Hedman, B.; Hodgson, K. O.; Solomon, E. I. Inorg. Chem. 1999, 38, 3676-3683.

(7) Shu, L.; Chiou, Y.-M.; Orville, A. M.; Miller, M. A.; Lipscomb, J. D.; Que, L., Jr. Biochemistry 1995, 34, 6649-6659.

(8) Brennan, B. A.; Chen, Q. H.; Juarezgarcia, C.; True, A. E.; O'Connor, C. J.; Que, L., Jr. Inorg. Chem. 1991, 30, 1937-1943. gained through the analysis of the $\mathrm{Fe} \mathrm{K}$ pre-edge region. ${ }^{12-14}$ The $1 \mathrm{~s} \rightarrow 3 \mathrm{~d}$ pre-edge is electric dipole forbidden, and thus its intensity reflects the less efficient electric quadrupole mechanism $\left(\sim 10^{2}<\right.$ electric dipole). The pre-edge features can also gain intensity through mixing with the electric dipole allowed $1 \mathrm{~s} \rightarrow 4 \mathrm{p}$ transition in a noncentrosymmetric environment. Thus, the intensity of the iron $\mathrm{K}$ pre-edge reflects the amount of metal $4 \mathrm{p}$ character in the d-manifold due to a lower symmetry of the iron site. ${ }^{13}$ This produces $\mathrm{d}^{n+1}$ final states for which electronelectron repulsion and ligand field splittings result in a series of multiplets that spread the pre-edge over several electronvolts. This is an indirect method, however, for probing the detailed electronic structure of the metal $3 \mathrm{~d}$ orbitals.

The iron L-edge has been less utilized, although it can provide more detailed electronic information. The $2 \mathrm{p} \rightarrow 3 \mathrm{~d} \mathrm{~L}$ pre-edge transition is electric dipole allowed and has approximately 10-

(9) Dong, Y. H.; Fujii, H.; Hendrich, M. P.; Leising, R. A.; Pan, G. F.; Randall, C. R.; Wilkinson, E. C.; Zang, Y.; Que, L., Jr.; Fox, B. G.; Kauffmann, K.; Münck, E. J. Am. Chem. Soc. 1995, 117, 2778-2792.

(10) Waychunas, G. A.; Brown, G. E., Jr.; Apted, M. J. Phys. Chem. Miner. 1986, $13,31-47$

(11) Hitchcock, A. P.; Wen, A. T.; Rühl, E. Chem. Phys. 1990, 147, 51-63.

(12) Randall, C. R.; Shu, L.; Chiou, Y.-M.; Hagen, K. S.; Ito, M.; Kitajima, N.; Lachicotte, R. J.; Zang, Y.; Que, L., Jr. Inorg. Chem. 1995, 34, 1036 1039.

(13) Westre, T. E.; Kennepohl, P.; DeWitt, J. G.; Hedman, B.; Hodgson, K. O.; Solomon, E. I. J. Am. Chem. Soc. 1997, 119, 6297-6314.

(14) Wilke, M.; Farges, F.; Petit, P.-E.; Brown, G. E., Jr.; Martin, F. Am. Mineral. 2001, 86, 714-730. 
fold higher intensity than the metal K pre-edge. Furthermore, the resolution at the L-edge is $3-5$ times better ${ }^{15}$ than the $\sim 1.4 \mathrm{eV}$ resolution of the iron K-edge. ${ }^{16}$ The result is an intense, feature-rich spectrum probing electronic states of primarily metal $3 \mathrm{~d}$ character. Thus, the iron L-edge spectrum is better suited for detailed analysis of the electronic composition of the $d$ orbitals in metalloproteins and model systems.

In a molecular orbital picture, the ground state of a metal complex is described as a linear combination of metal and ligand valence orbitals. The normalized ground-state wave function may be written as

$$
\Psi=\left(1-\alpha^{2}\right)^{1 / 2}\left|M_{3 \mathrm{~d}}\right\rangle+\alpha\left|L_{n \mathrm{p}}\right\rangle
$$

where $M_{3 \mathrm{~d}}$ are the metal $3 \mathrm{~d}$ orbitals, $L_{n \mathrm{p}}$ are the ligand valence $\mathrm{p}$ orbitals, and $\alpha^{2}$ reflects the covalency of the metal-ligand interaction. As the L-edge excitation originates from a metalcentered $2 \mathrm{p}$ orbital, only transitions to the metal-centered component of the ground state wave function have intensity. Thus, the metal L pre-edge transition probes the metal $3 \mathrm{~d}$ manifold, and the integrated intensity of the L-edge X-ray absorption spectrum can be directly related to the covalency $\left(1-\alpha^{2}\right)$ of the partially and/or fully unoccupied d orbitals in a metal complex. This methodology has previously been used to experimentally determine the covalency of the $\mathrm{Cu}$-ligand bonds in certain blue copper ${ }^{17}$ and $\mathrm{Cu}_{\mathrm{A}}$ proteins ${ }^{18}$ and model complexes, which are missing a single electron in an otherwise filled d manifold. ${ }^{19-21}$ For iron complexes, this methodology is complicated by the fact that there are 4 or 5 unoccupied or half-occupied d orbitals for Fe(II) and Fe(III), respectively. In a ligand field, the d orbitals split into symmetry sets of orbitals that have differing covalencies. The integrated intensity thus reflects the sum of the covalencies for the different partially occupied d orbitals (e.g., $\mathrm{t}_{2 \mathrm{~g}}$ and $\mathrm{e}_{\mathrm{g}}$ sets in $O_{h}$ ).

Besides the multiple unoccupied orbitals with differing covalencies, there are a number of multiplet and shake-up states in the $\mathrm{Fe}^{\mathrm{II}}$ or $\mathrm{Fe}^{\mathrm{III}}$ L-edge that further complicate the experimental spectrum. As in systems with a singly unoccupied $3 \mathrm{~d}$ valence orbital such as copper, the primary effect in the L-edge spectrum is due to the coupling of the spin and angular momentum of the $2 p$ core hole in the excited state. This $2 p$ spin-orbit coupling $\left(\lambda_{L \cdot S}\right)$ splits the L-edge into two features, the lower energy $\mathrm{L}_{3}$-edge corresponding to the $2 \mathrm{p}_{3 / 2}$ final state, and the $\mathrm{L}_{2}$-edge corresponding to the $2 \mathrm{p}_{1 / 2}$ final state at $3 / 2 * \lambda_{L} \cdot S$ (12.4 eV for iron) higher in energy. For iron complexes with multiple holes in the ground state, additional effects including the $\mathrm{d}^{n+1}$ final state electron repulsion and ligand field splitting,

(15) Cramer, S. P.; deGroot, F. M. F.; Ma, Y.; Chen, C. T.; Sette, F.; Kipke, C. A.; Eichhorn, D. M.; Chan, M. K.; Armstrong, W. H.; Libby, E.; Christou, G.; Brooker, S.; Mckee, V.; Mullins, O. C.; Fuggle, J. C. J. Am. Chem. Soc. 1991, 113, 7937-7940.

(16) Lytle, F. W. In Applications of Synchrotron Radiation; Winick, H., Xiam, D., Ye, M.-H., Huang, T., Eds.; Gordon and Breach Science Publishers: New York, 1989; pp 135-223.

(17) George, S. J.; Lowery, M. D.; Solomon, E. I.; Cramer, S. P. J. Am. Chem. Soc. 1993, 115, 2968-2969.

(18) George, S. D.; Metz, M.; Szilagyi, R. K.; Wang, H. X.; Cramer, S. P.; Lu, Y.; Tolman, W. B.; Hedman, B.; Hodgson, K. O.; Solomon, E. J. Am. Chem. Soc. 2001, 123, 5757-5767.

(19) Randall, D. W.; George, S. D.; Hedman, B.; Hodgson, K. O.; Fujisawa, K.; Solomon, E. I. J. Am. Chem. Soc. 2000, 122, 11620-11631.

(20) Randall, D. W.; George, S. D.; Holland, P. L.; Hedman, B.; Hodgson, K. O.; Tolman, W. B.; Solomon, E. I. J. Am. Chem. Soc. 2000, 122, 1163211648 .

(21) Basumallick, L.; George, S. D.; Randall, D. W.; Hedman, B.; Hodgson, K. O.; Fujisawa, K.; Solomon, E. I. Inorg. Chim. Acta 2002, 337, 357365 . the $d-p$ electron repulsion, and the spin-orbit coupling of the $3 \mathrm{~d}$ orbitals also modify the spectrum, spreading the $\mathrm{Fe}^{\mathrm{II}}$ or $\mathrm{Fe}^{\mathrm{III}}$ L-edge over an energy range of $\sim 20 \mathrm{eV}$. An L-edge ligand field multiplet model has been developed by Thole and co-workers to allow a quantitative determination of these states and provide a basic understanding of the multiplet structure in an L-edge spectrum. ${ }^{22-24}$

However, as seen below, application of the ligand field theory-multiplet model does not provide an adequate fit to the L-edge spectra of many iron complexes. The model accounts for covalency only in terms of an atomic Slater integral reduction parameter, $\kappa$, comparable to the nephelauxetic effect, $\beta$, used in optical spectroscopy. In most cases, ligand-to-metal charge transfer (LMCT) satellite features, which are not included in the ligand field model, are also apparent in the L-edge spectrum. Extension of the multiplet model to include charge transfer (CT) allows for analysis of the L-edge spectrum with covalency included as a consequence of LMCT. ${ }^{25,26}$ Application of this charge transfer multiplet model results in simulated intensity in the CT region and a better fit to the data. When the charge transfer model is used to simulate the experimental multiplet spectrum and adapted to include differential orbital covalency (DOC), defined here as the difference in the metal d character of the $t_{2 g}$ and $e_{g}$ orbitals, a detailed fit to the L-edge spectrum can be obtained, allowing for the analysis of previously inaccessible highly covalent sites.

In this study, the ligand field multiplet model has been applied to a series of ferric and ferrous non-heme iron complexes. ${ }^{27}$ In most ferric cases, this model does not adequately reproduce the spectral features, especially with respect to charge-transfer satellite features $\sim 5 \mathrm{eV}$ above the main $\mathrm{L}_{3}$-edge multiplets. The application of the charge transfer model is therefore required to fit the L-edge spectra of these complexes. The results of the fit are used in a projection method developed below, wherein the ground state wave functions used to simulate the L-edge spectrum are projected into a set of final states for which multiplet effects have been eliminated. This allows for a direct measurement of the different contributions of LMCT pathways to the $t_{2 g}$ and $e_{g}$ symmetry subsets of the $d$ manifold and, therefore, the DOC. The resulting experimentally determined DOC is compared with theoretically calculated values from density functional theory and chemical trends over a series of high- and low-spin ferric and ferrous complexes. The utility of this method toward problems in bioinorganic chemistry is discussed.

\section{Experimental Procedures}

2.1. Sample Preparation. Samples, prepared as previously described, ${ }^{28-34}$ were finely ground and spread across double-sided adhesive

(22) de Groot, F. M. F.; Fuggle, J. C.; Thole, B. T.; Sawatzky, G. A. Phys. Rev. B. 1990, 41, 928-937.

(23) de Groot, F. M. F.; Fuggle, J. C.; Thole, B. T.; Sawatzky, G. A. Phys. Rev. B. 1990, 42, 5459-5468.

(24) Thole, B. T.; van der Laan, G.; Butler, P. H. Chem. Phys. Lett. 1988, 149, $295-299$

(25) de Groot, F. M. F. J. Electron Spectrosc. Relat. Phenom. 1994, 67, 529622.

(26) Okada, K.; Kotani, A.; Thole, B. T. J. Electron Spectrosc. Relat. Phenom. 1992, 58, 325-343.

(27) Abbreviations used: acac $=$ acetylacetonate; ox $=$ oxalate; ida $=$ iminodiacetic acid; tacn = 1,4,7-triazacyclononane.

(28) Iball, J.; Morgan, C. H. Acta Crystallogr. 1967, 23, 239-244.

(29) Kistenmacher, T. J.; Stucky, G. D. Inorg. Chem. 1968, 7, 2150-2155.

(30) Lauher, J. W.; Ibers, J. A. Inorg. Chem. 1975, 14, 348-352

(31) Beattie, J. K.; Moore, C. J. Inorg. Chem. 1982, 21, 1292-1295.

(32) Clark, H. C.; Curtis, N. F.; Odell, A. L. J. Chem. Soc. 1954, 63-66. 
conductive graphite tape attached to a copper paddle. The paddle was affixed to a rotary stage and inserted into the experimental chamber aligned at $45^{\circ}$ to the incident beam. For all air-sensitive samples, samples were applied to the adhesive tape on the copper paddle in an oxygen free $(<1 \mathrm{ppm})$ glovebox, and the paddle was affixed to the rotary stage in an $\mathrm{N}_{2}$-purged glovebag immediately before insertion into the sample chamber.

2.2. XAS Data Collection. X-ray absorption spectra were recorded at the Stanford Synchrotron Radiation Laboratory on the 31-pole wiggler beam line 10-1 under ring operating conditions of $50-100 \mathrm{~mA}$ and 3 $\mathrm{GeV}$. The radiation was monochromatized using a spherical grating monochromator set at 1000 lines $/ \mathrm{mm}$ and $20 \mu \mathrm{m}$ entrance and exit slits. Sample measurement was performed using total electron yield mode where the sample signal $\left(I_{1}\right)$ was collected with a Galileo 4716 channeltron electron multiplier aligned to $45^{\circ}$ relative to the copper paddle and $90^{\circ}$ to the incident beam. The signal was flux normalized $\left(I_{1} / I_{0}\right)$ by the photocurrent of a gold-grid reference monitor $\left(I_{0}\right)$. Data for all samples were recorded in a sample chamber maintained at $<1 \times 10^{-5}$ Torr, isolated from the UHV beam line by a $1000 \AA$ diamond window. The photon energy was two-point calibrated using $\mathrm{Fe}_{2} \mathrm{O}_{3}$ as a reference prior to and after collecting data from each sample, aligning the maximum intensity of the $\mathrm{Fe}_{2} \mathrm{O}_{3} \mathrm{~L}_{3}$-edge to $708.5 \mathrm{eV}$ and the lower-energy $\mathrm{L}_{2}$ feature to $720.1 \mathrm{eV}$. The variance in the calibration energies prior to and after each sample was used to linearly shift the experimental spectra between calibration scans. In all cases, the maximum calibration shift between $\mathrm{Fe}_{2} \mathrm{O}_{3}$ scans before and after sample data collection was $\leq 0.4 \mathrm{eV}$. A number of scans (typically $4-6$ ) were collected at room temperature for each sample to monitor spectrum reproducibility. Data were collected over the energy range 670-830 $\mathrm{eV}$ to adequately normalize each data set after the maximum of the $2 \mathrm{p}$ photoabsorption cross-section.

2.3. XAS Multiplet Calculations. Atomic multiplet calculations were performed using the software suite developed by R. D. Cowan and co-workers ${ }^{35}$ and modified by B. T. Thole. The atomic Hamiltonian can be written in the form

$$
\begin{gathered}
H=H_{\mathrm{ave}}+H_{l \mathrm{~s}}+H_{\mathrm{ee}} \\
H_{\mathrm{ave}}=\sum_{N} \frac{p_{i}^{2}}{2 m}+\sum_{N} \frac{-Z e^{2}}{r_{i}} \\
H_{l s}=\sum_{N} \xi\left(r_{i}\right) l_{i} \cdot s_{i} \\
H_{\mathrm{ee}}=\sum_{i, j} \frac{e^{2}}{r_{i j}}
\end{gathered}
$$

$H_{\text {ave }}$ is the average energy of the configuration and contains the kinetic term and the interactions of the electrons with the nucleus; $H_{l s}$ represents the spin-orbit coupling of the electrons; and $H_{\mathrm{ee}}$ is the electronelectron interaction term, whose solutions produce the well-known Slater-Condon-Shortley parameters $F_{i}$ (ground and excited states) and $G_{i}$ (excited-state only). A Hartree-Fock calculation is performed to calculate radial wave functions that are integrated to produce the radial part of the matrix elements. Coefficient matrices for atomic symmetry are then computed, reading the atomic parameter values and calculating and diagonalizing the energy matrices to obtain energy levels and eigenvectors denoted by atomic term symbols of the form ${ }^{2 S+1} L_{J}$.

The ligand field multiplet (LFM) model extends the atomic Hamiltonian with an electrostatic field surrounding the metal center. The

(33) Wieghardt, K.; Schmidt, W.; Herrmann, W.; Küppers, H.-J. Inorg. Chem. 1983, 22, 2953-2956.

(34) Hatfield, W. E.; Fay, R. C.; Pfluger, C. E.; Piper, T. S. J. Am. Chem. Soc. 1963, 85, 265-269.

(35) Cowan, T. The Theory of Atomic Structure and Spectra; University of California Press: Berkeley, CA, 1981. potential field is treated as a perturbation $\left(H_{\mathrm{LF}}\right)$ on the atomic Hamiltonian and serves to split or branch the atomic configurations (as developed by Butler). ${ }^{36}$

For the LFM section of this study, the Slater-Condon-Shortley parameters (all $F_{i}$ and $G_{i}$ ) are first reduced to $80 \%$ of the Hartre-Fock calculated values to account for the excessive electron-electron repulsion found in these calculations of the free ion, further reduced according to literature values of $\beta$ for the complexes, and finally modified in a stepwise manner from this starting point to find a best simulation. Similarly, $10 D q$ is set initially to literature values and modified in a stepwise manner to obtain the best simulation of the data.

Charge transfer effects have been included as an additional perturbation to the Hamiltonian ${ }^{26}$ in such cases that the LFM model is insufficient to simulate the data. The LFM model uses a single configuration for which the effective atomic and ligand field Hamiltonians are solved:

$$
\begin{gathered}
H_{\mathrm{GS}, 1}=\left\langle 3 d^{n}\left|H_{\mathrm{ave}}+H_{l s(d)}+H_{\mathrm{ee}}+H_{\mathrm{LF}}\right| 3 d^{n}\right\rangle \\
H_{\mathrm{ES}, 1}=\left\langle\underline{c} 3 d^{n+1}\left|H_{\mathrm{ave}}+H_{l s(d)}+\mathrm{H}_{l s(p)}+H_{\mathrm{ee}}+H_{\mathrm{LF}}\right| \underline{c} 3 d^{n+1}\right\rangle
\end{gathered}
$$

Charge transfer effects associated with covalency (vida infra) are included by adding a second configuration to the XAS ground (and final) state at an energy $\Delta$ (and $\Delta^{\prime}$ ) above the first configuration.

$$
\begin{gathered}
H_{\mathrm{GS}, 2}=\left\langle 3 d^{n+1} \underline{L}\left|H_{\mathrm{ave}}+H_{l s(d)}+H_{\mathrm{ee}}+H_{\mathrm{LF}}\right| 3 d^{n+1} \underline{L}\right\rangle \\
H_{\mathrm{ES}, 2}=\left\langle\underline{c} 3 d^{n+2} \underline{L}\left|H_{\mathrm{ave}}+H_{l s(d)}+H_{l s(p)}+H_{\mathrm{ee}}+H_{\mathrm{LF}}\right| \underline{c} 3 d^{n+2} \underline{L}\right\rangle
\end{gathered}
$$

The two initial (and final) state configurations are coupled by configuration interaction, governed by the mixing parameter $T$. Thus, for the ground state

$$
T=\left\langle 3 d^{n}|h| 3 d^{n+1} \underline{\mathrm{L}}\right\rangle
$$

where $h$ is the molecular Hamiltonian.

This charge transfer mixing leads to the presence of satellite features in L-edge XAS spectra. The intensities of the multiplet features are given by the one-electron electric dipole allowed $\mathrm{p} \rightarrow \mathrm{d}$ transition probability contained in each many-electron wave function. This intensity is then redistributed over the main peak and the satellite final states. The intensities of the main and satellite peaks are then proportional to $\left(\alpha_{1} \alpha_{2}+\beta_{1} \beta_{2}\right)^{2}$ and $\left(\alpha_{1} \beta_{2}-\beta_{1} \alpha_{2}\right)^{2}$, respectively, where the bonding ground state $\left(\Psi_{\mathrm{GS}, \mathrm{B}}\right)$ wave function and bonding and antibonding excited state $\left(\Psi_{\mathrm{ES}, B}\right.$ and $\left.\Psi_{\mathrm{ES}, A B}\right)$ wave functions can be written as

$$
\begin{aligned}
\Psi_{\mathrm{GS}, B}=\alpha_{1}\left|3 d^{n}\right\rangle+\beta_{1}\left|3 d^{n+1} \underline{\mathrm{L}}\right\rangle \quad & \Psi_{\mathrm{ES}, B}=\alpha_{2}\left|3 d^{n}\right\rangle+\beta_{2}\left|3 d^{n+1} \underline{\mathrm{L}}\right\rangle \\
& \Psi_{\mathrm{ES}, A B}=\beta_{2}\left|3 d^{n}\right\rangle-\alpha_{2}\left|3 d^{n+1} \underline{\mathrm{L}}\right\rangle
\end{aligned}
$$

The main XAS features correspond to the $\Psi_{G S, B} \rightarrow \Psi_{E S, B}$ transitions and the satellite peaks correspond to the $\Psi_{G S, B} \rightarrow \Psi_{E S, A B}$ transitions.

The absolute transition intensities cannot be quantitatively estimated from either the calculation or the experiment. Therefore, to directly compare experimental and theoretical spectral intensities, the simulated spectra are renormalized to correlate to the experimental data. For the ligand field simulations, which do not include covalency explicitly, the simulated spectrum is renormalized to a total integrated intensity of $100 \%$ metal d contribution to the wave function, based on the integrated intensity of (and thus metal d contribution to) the experimental spectrum. For the isotropic and differential orbital covalency simulations, the simulated intensity has been renormalized to match the integrated intensity of the experimental spectrum, since the charge

(36) Butler, P. H. Point Group Symmetry: Applications, Methods and Tables; Plenum Press: New York, 1981. 
transfer multiplet model used for these simulations does include covalency in terms of the charge transfer configurations $3 \mathrm{~d}^{n+1} \mathrm{~L}$.

2.4. Density Functional Calculations. Molecular orbital calculations were based on approximate density functional theory (DFT) using the Amsterdam density functional computational package (ADF, version 2000). ${ }^{37,38}$ All DFT calculations were performed using the local density approximation (LDA) parametrization of Vosko, Wilk, and Nussair ${ }^{39}$ with nonlocal gradient corrections of Becke ${ }^{40-42}$ and Perdew ${ }^{43,44}$ added self-consistently. The frozen core approximation was used for the $1 \mathrm{~s}$ orbitals of second row elements and for the $1 \mathrm{~s}, 2 \mathrm{~s}$, and $2 \mathrm{p}$ orbitals of chlorine and iron. All valence orbitals were described using triple- $\zeta$ Slater type orbital (STO) basis functions (basis set IV). Spin-unrestricted calculations were refined with a numerical integration precision of 5.0 and to a final gradient of no greater than $10^{-6}$ au. Orbital contributions to calculated molecular orbitals are reported as Mulliken populations to the nearest $0.1 \%$.

\section{Results}

Most iron L-edge studies to date have recorded data over a limited energy range, typically ending around $730 \mathrm{eV} \cdot{ }^{24,45-49}$ While this is adequate to describe the $2 \mathrm{p} \rightarrow 3 \mathrm{~d}$ pre-edge multiplet features, normalization of the data for quantitative comparison of intensities requires the spectral region to be extended beyond the delayed $2 \mathrm{p}$ absorption maximum at $\sim 740 \mathrm{eV}$. Thus, the data presented here have been recorded from 670 to $830 \mathrm{eV}$. From the averaged raw data, a linear background was fit to the preedge region $(<700 \mathrm{eV})$ and subtracted from the entire spectrum. Normalization of the data was accomplished by fitting a straight line to the postedge region $(770-830 \mathrm{eV})$ and normalizing the edge jump to 1.0 at $830 \mathrm{eV}$. Two arctangent functions were used to model the $2 \mathrm{p}_{3 / 2} \rightarrow$ continuum and $2 \mathrm{p}_{1 / 2} \rightarrow$ continuum ionization edges. The slope of each of the arctangents was determined by fitting the experimental $2 p$ absorption cross section ${ }^{50}$ to an arctangent function with varied amplitude and slope. The two arctangents were separated in energy by $3 / 2 * \lambda_{L} \cdot S$ $(12.4 \mathrm{eV})$ and fixed with an intensity ratio of $2: 1$ for the $\mathrm{L}_{3^{-}}$ and $\mathrm{L}_{2}$-edge, respectively. The absolute energy of the arctangent modeling the $\mathrm{L}_{3}$-edge was determined from a critical evaluation of calibrated XPS data on complexes of similar oxidation and spin states to the sample of interest. ${ }^{51-54}$ For high-spin ferric and ferrous complexes, the $\mathrm{L}_{3}$ arctangents were centered at 713.6

(37) te Velde, G.; Bickelhaupt, F. M.; Baerends, E. J.; Guerra, C. F.; van Gisbergen, S. J. A.; Snijders, J. G.; Ziegler, T. J. Comput. Chem. 2001, $22,931-967$

(38) Baerends, E. J.; Ellis, D. E.; Ros, P. Chem. Phys. 1973, 2, 41-51.

(39) Vosko, S. J.; Wilk, M.; Nussair, M. Can. J. Phys. 1980, 58, 1200-1211.

(40) Becke, A. J. Chem. Phys. 1986, 84, 4524-4529.

(41) Becke, A. J. Chem. Phys. 1988, 88, 1053-1062.

(42) Becke, A. Phys. Rev. A 1988, 38, 3098-3100.

(43) Perdew, J. P. Phys. Rev. B. 1986, 34, 7406.

(44) Perdew, J. P. Phys. Rev. B. 1986, 33, 8822-8824.

(45) Atanasov, M.; Potze, R. H.; Sawatzky, G. A. J. Solid State Chem. 1995, $119,380-393$.

(46) Schofield, P. F.; van der Laan, G.; Henderson, C. M. B.; Cressey, G. Mineral. Mag. 1998, 62, 65-75.

(47) Wang, H.; Peng, G.; Miller, L. M.; Scheuring, E. M.; George, S. J.; Chance, M. R.; Cramer, S. P. J. Am. Chem. Soc. 1997, 119, 4921-4928.

(48) George, S. J.; van Elp, J.; Chen, J.; Ma, Y.; Chen, C. T.; Park, J.-B.; Adams, M. W. W.; Searle, B. G.; de Groot, F. M. F.; Fuggle, J. C.; Cramer, S. P J. Am. Chem. Soc. 1992, 114, 4426-4427.

(49) Arrio, M.-A.; Sainctavit, P.; Cartier dit Moulin, C.; Mallah, T.; Verdaguer, M.; Pellegrin, E.; Chen, C. T. J. Am. Chem. Soc. 1996, 118, 8, 64226427.

(50) Yeh, J. J.; Lindau, I. At. Data Nucl. Data Tables 1985, 32, 1-155.

(51) Fujiwara, M.; Matsushita, T.; Ikeda, S. X-sen Bunseki no Shinpo 1995, 26, 263-272.

(52) Srivastava, S.; Badrinarayanan, S.; Mukhedkar, A. J. Polyhedron 1985, 4, 409-414.

(53) Inoue, V. H.; Fluck, E.; Binder, H.; Yanagisawa, S. Z. Anorg. Allg. Chem. 1981, 483, 75-85.

(54) Kennepohl, P.; Solomon, E. I. Inorg. Chem. 2003, 42, 679-688.

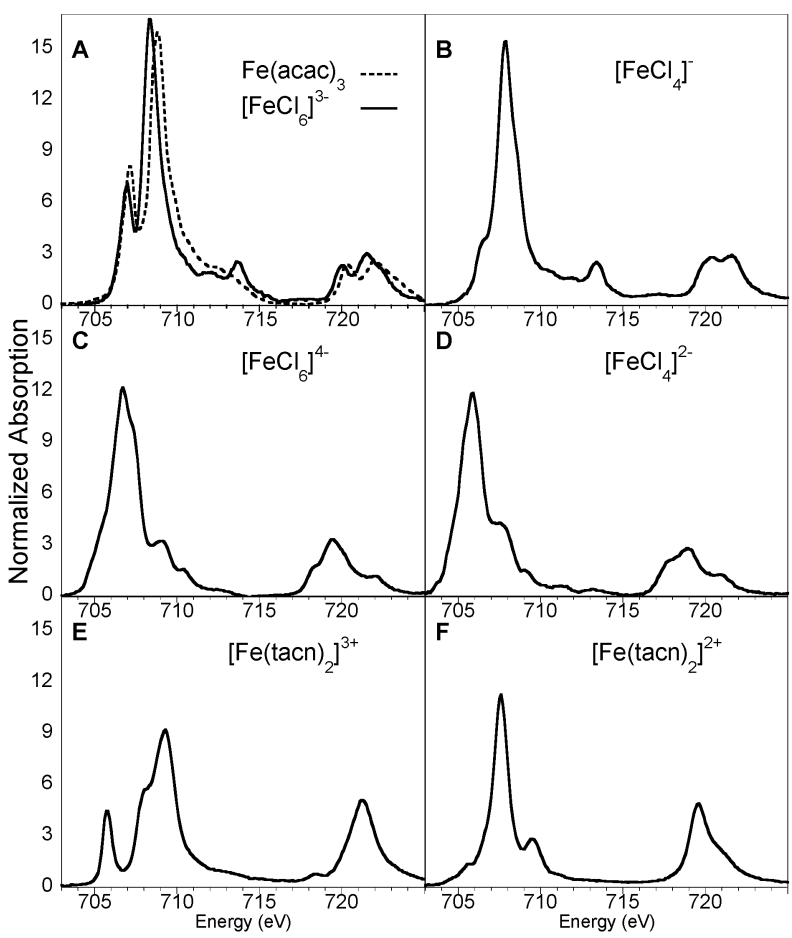

Figure 1. L-edge multiplets and integrated intensities for selected complexes: (A) $\left[\mathrm{FeCl}_{6}\right]^{3-}$ (-) and $\mathrm{Fe}(\mathrm{acac})_{3}(---)$; (B) $\left[\mathrm{FeCl}_{4}\right]^{-}$; (C) $\left[\mathrm{FeCl}_{6}\right]^{4-}$; (D) $\left[\mathrm{FeCl}_{4}\right]^{2-}$; (E) $\left[\mathrm{Fe}(\operatorname{tacn})_{2}\right]^{3+}$; (F) $\left[\mathrm{Fe}(\mathrm{tacn})_{2}\right]^{2+}$.

and $710.8 \mathrm{eV}$, respectively, whereas, for low-spin ferric and ferrous complexes, they were centered at 709.9 and $708.5 \mathrm{eV}$, respectively. Subtracting the arctangents from the data results in normalized $2 \mathrm{p} \rightarrow \Psi^{*}$ multiplet spectra without intensity contributions from the ionization edges. The integrated intensity (over the range 700-730 eV) of the resulting multiplet spectra was thus used to quantitatively evaluate the d-orbital character in the ground state wave functions.

Several characteristic features are clearly visible among the representative L-edge spectra shown in Figure 1. The data from high-spin ferric octahedral complexes (Figure 1A) are similar to those previously published for monomeric and dimeric highspin ferric complexes, ${ }^{55,56}$ characterized by two strong features in the $\mathrm{L}_{3}$-edge at $\sim 707$ and $\sim 709 \mathrm{eV}$, followed by small multiplet and satellite features $(710-715 \mathrm{eV})$ and two relatively weak features in the $\mathrm{L}_{2}$-edge $(719-725 \mathrm{eV})$. It should be noted that an additional feature appears at $714 \mathrm{eV}$ of the $\mathrm{L}_{3}$-edge in the octahedral $\left[\mathrm{FeCl}_{6}\right]^{3-}$ complex (Figure 1A, solid line) that does not appear in the other ferric high-spin octahedral complexes. Similar to previously published data, ${ }^{56}$ the $\left[\mathrm{FeCl}_{4}\right]^{-}$ complex (Figure 1B) exhibits a single strong $\mathrm{L}_{3}$ feature at 708 $\mathrm{eV}$ and smaller multiplet/satellite features from 710 to $715 \mathrm{eV}$, followed by the $\mathrm{L}_{2}$-edge consisting of two features at 720 and $722 \mathrm{eV}$ split to a smaller degree than in the high-spin ferric $O_{h}$ case. A somewhat sharp feature at $\sim 713 \mathrm{eV}$ is also observed, similar to the $714 \mathrm{eV}$ feature in the $\left[\mathrm{FeCl}_{6}\right]^{3-}$ complex. For high-spin ferrous complexes, only subtle differences are observed between the octahedral $\left(\left[\mathrm{FeCl}_{6}\right],{ }^{4-}\right.$ Figure $\left.1 \mathrm{C}\right)$ and tetrahedral $\left(\left[\mathrm{FeCl}_{4}\right]^{2-}\right.$, Figure $\left.1 \mathrm{D}\right)$ model complexes: the multiplet features to the high-energy side of the $\mathrm{L}_{3}$ peak (from

(55) Peng, G.; van Elp, J.; Jang, H.; Que, L., Jr.; Armstrong, W. H.; Cramer, S. P. J. Am. Chem. Soc. 1995, 117, 2515-2519.

(56) Cartier, C.; Flank, A. M. In X-ray Absorption Fine Structure; Hasnain, S. S., Ed.; E. Horwood Ltd.: New York, 1991; pp 659-661. 
Table 1. Summary of L-edge Integrated Intensities, DOC Simulation Results, and Molecular Orbital Calculations

\begin{tabular}{|c|c|c|c|c|c|c|c|c|c|c|c|}
\hline & & \multicolumn{6}{|c|}{ L-edge covalency } & \multicolumn{4}{|c|}{ DFT (BP86) } \\
\hline & & intensity & avg & $\langle d M\rangle$ & $\mathrm{Fe}\left(\mathrm{t}_{2}\right)$ & $\mathrm{Fe}(\mathrm{e})$ & DOC & avg & $\mathrm{Fe}\left(\mathrm{t}_{2}\right)$ & $\mathrm{Fe}(\mathrm{e})$ & $\mathrm{DOC}$ \\
\hline$\left[\mathrm{FeCl}_{4}\right]^{-}$ & $\mathrm{Fe}^{\mathrm{III}} \mathrm{hs} T_{d}$ & 50.5 & 81 & 4.05 & 76 & 89 & -13 & 71 & 68 & 77 & -9 \\
\hline$\left[\mathrm{FeCl}_{6}\right]^{3-}$ & $\mathrm{Fe}^{\mathrm{III}}$ hs $O_{h}$ & 53.1 & 85 & 4.25 & 95 & 70 & 25 & 76 & 85 & 64 & 21 \\
\hline$\left[\mathrm{FeCl}_{4}\right]^{2-}$ & $\mathrm{Fe}^{\mathrm{II}} \mathrm{hs} T_{d}$ & 43.9 & 90 & 5.40 & 87 & 93 & -6 & 86 & 84 & 89 & -5 \\
\hline$\left[\mathrm{FeCl}_{6}\right]^{4-}$ & $\mathrm{Fe}^{\mathrm{II}}$ hs $O_{h}$ & 44.7 & 92 & 5.52 & 96 & 88 & 8 & 88 & 94 & 83 & 11 \\
\hline $\mathrm{Fe}(\mathrm{acac})_{3}$ & $\mathrm{Fe}^{\mathrm{III}}$ hs $O_{h}$ & 52.2 & 83 & 4.15 & 90 & 73 & 17 & 77 & 83 & 68 & 15 \\
\hline$\left[\mathrm{Fe}(\mathrm{ox})_{3}\right]^{3-}$ & $\mathrm{Fe}^{\mathrm{III}}$ hs $O_{h}$ & 52.3 & 84 & 4.20 & 90 & 76 & 14 & 78 & 83 & 72 & 11 \\
\hline$\left[\mathrm{Fe}(\mathrm{ida})_{2}\right]^{-}$ & $\mathrm{Fe}^{\mathrm{III}}$ hs $O_{h}$ & 51.2 & 82 & 4.10 & 88 & 72 & 16 & 77 & 83 & 68 & 15 \\
\hline$\left[\mathrm{Fe}(\operatorname{tacn})_{2}\right]^{3+}$ & $\mathrm{Fe}^{\mathrm{III}}$ ls $O_{h}$ & 40.6 & 70 & 3.50 & 99 & 63 & 36 & 68 & 93 & 62 & 31 \\
\hline$\left[\mathrm{Fe}(\operatorname{tacn})_{2}\right]^{2+}$ & $\mathrm{Fe}^{\mathrm{II}}$ ls $O_{h}$ & 37.4 & 72 & 4.32 & & 72 & 0 & 71 & & 71 & 0 \\
\hline
\end{tabular}

718 to $720 \mathrm{eV}$ ) are slightly perturbed relative to one another, and there is a minor difference in intensity in the low-energy shoulder found on the $\mathrm{L}_{2}$ peak at $718 \mathrm{eV}$. The high-spin ferrous complexes are, however, quite different in comparison to the corresponding high-spin ferric complexes. Most striking is the lack of a second, high-intensity feature in the $\mathrm{L}_{3}$-edge of $\left[\mathrm{FeCl}_{6}\right]^{4-}$ relative to the ferric complex, reflecting a decrease in multiplet splitting, likely due to the reduced ligand field strength and lowered covalency of the complex (vide infra). Comparing the ferric and ferrous tetrahedral chloride complexes (Figure 1B and D, respectively), a redistribution of intensity is clearly evident. The ferrous complex lacks the sharp absorption feature at $\sim 713 \mathrm{eV}$ of the ferric complex, and the $\mathrm{L}_{2}$ multiplet features are spread relative to those of the ferric complex.

Ferric and ferrous low-spin $\sigma$-donor complexes with simple ligands are markedly different from each other and from their high-spin counterparts. The $\mathrm{L}_{3}$-edge of $\left[\mathrm{Fe}(\operatorname{tacn})_{2}\right]^{3+}$, a lowspin ferric complex (Figure 1E) is dominated by a strong, sharp absorption at $\sim 706 \mathrm{eV}$ corresponding to a transition to the single half-occupied orbital of $t_{2 \mathrm{~g}}$ symmetry $\left(\mathrm{a}\left(\mathrm{t}_{2 \mathrm{~g}}\right)^{6}\right.$ final state) in the d-manifold and a broader, more intense feature to higher energy from 707 to $712 \mathrm{eV}$ from the $\mathrm{t}_{2 \mathrm{~g}} \mathrm{e}_{\mathrm{g}}{ }^{1}$ excited-state multiplets. The feature corresponding to the $t_{2}$ hole in the $\mathrm{L}_{2}$-edge at 718 $\mathrm{eV}$ is significantly diminished in amplitude relative to the $\mathrm{L}_{3} \mathrm{t}_{2}$ feature, followed by a single broad $\mathrm{L}_{2}$ absorption envelope from 720 to $725 \mathrm{eV}$. Alternatively, low-spin ferrous complexes lack the electron-hole in the $t_{2 \mathrm{~g}}$ orbitals. Thus, the $\mathrm{L}_{3}$ - and $\mathrm{L}_{2}$-edges of $\left[\mathrm{Fe}(\operatorname{tacn})_{2}\right]^{2+}$ (Figure 1F) exhibit only a single strong absorption band at $\sim 707$ and $720 \mathrm{eV}$, respectively, followed by a smaller multiplet peak separated by $\sim 1-2 \mathrm{eV}$. This is similar to a previously published data $^{57}$ for a mixed ligand lowspin ferrous system.

The integrated intensities for specific complexes (Figure 1 and Figures S1-S3, Supporting Information) are given in the first column of Table 1. In general, ferrous complexes exhibit a lower overall intensity than the corresponding ferric complexes. The L-edge spectrum of $\left[\mathrm{FeCl}_{6}\right]^{3-}$ has a total integrated intensity of 53.1 units, quite similar to those of $\mathrm{Fe}(\mathrm{acac})_{3}$ and $\left[\mathrm{FeCl}_{4}\right]^{-}$with 52.2 and 50.5 units of integrated intensity, respectively. The ferrous $O_{h}$ and $T_{d}$ chloride complexes have only 44.7 and 43.9 units of integrated intensity, respectively. The low-spin octahedral $\left[\mathrm{Fe}(\operatorname{tacn})_{2}\right]^{2 / 3+}$ complexes exhibit L-edge spectra with a much lower intensity than octahedral highspin complexes of the same oxidation state. The intensity maximum in the spectrum of $\left[\mathrm{Fe}(\operatorname{tacn})_{2}\right]^{3+}$ (Figure 1E) is much lower than that of $\left[\mathrm{FeCl}_{6}\right]^{3-}$ (Figure $1 \mathrm{~A}$ ) with a correspondingly

(57) Cartier dit Moulin, C.; Rudolf, P.; Flank, A.-M.; Chen, C.-T. J. Phys. Chem. 1992, 96, 6196-6198 decreased integrated intensity of 40.6 normalized units. [Fe$\left.(\operatorname{tacn})_{2}\right]^{2+}$ (Figure 1F) has an integrated intensity of 37.4 units, significantly less than that of $\left[\mathrm{FeCl}_{6}\right]^{4-}$ (Figure 1C) but similar to that of $\left[\mathrm{Fe}(\operatorname{tacn})_{2}\right]^{3+}$.

\section{Analysis}

4.1. Total Covalency. The metal L-edge spectrum may consist of transitions from the metal $2 p$ orbital to any of the metal valence $3 \mathrm{~d}, 4 \mathrm{~s}$, and $4 \mathrm{p}$ orbitals. However, from the metal $2 \mathrm{p}$ orbitals, only the metal $3 \mathrm{~d}$ and $4 \mathrm{~s}$ orbitals are accessible by electric dipole allowed transitions. Furthermore, the $2 p \rightarrow 4 s$ transition is $\sim 20$-fold lower in intensity and consequently can be neglected. Hence, only the $2 \mathrm{p} \rightarrow 3 \mathrm{~d}$ contribution to the metal $\mathrm{L}$ pre-edge needs to be considered for complexes of most firsttransition row elements. Covalent mixing of the $3 \mathrm{~d}$ orbitals with the ligand np orbitals will decrease the amount of iron $3 \mathrm{~d}$ character in each of the allowed final state wave functions $\left(1-\alpha^{2}\right.$ in eq 1.1) and thus proportionally decrease the electricdipole intensity for each multiplet transition. Multiplet effects distribute the L-edge spectral transitions across a range of $\sim 20$ $\mathrm{eV}$, where each transition consists of varying amounts of metal $3 \mathrm{~d}$ and ligand $n \mathrm{p}$ character. The total metal L-pre-edge transition intensity therefore is proportional to the amount of metal $d$ character summed over the different final state wave functions. The metal $3 \mathrm{~d}$ character obtained from independent spectroscopic methods can then be used to calibrate the integrated intensity from Fe L-edge XAS. Ferric tetrachloride has been extensively studied; the covalency of the metal-ligand bond has been determined from the ligand K-edge XAS spectrum ${ }^{58}$ and corresponds to $80.2 \%$ metal d character averaged over the five half-occupied $3 \mathrm{~d}$ orbitals. Thus we can calibrate $80.2 \%$ metal $\mathrm{d}$ character to 50.5 units of L-edge intensity (Table 1 ) or $\sim 1.6 \%$ metal $d$ character per unit of L-edge intensity in each of the five half-occupied d orbitals and a total covalency of $7.9 \%$ metal d-character. This correlates well with past $\mathrm{Cu}$ L-edge results ${ }^{17}$ that found a total of $7.2 \% \mathrm{Cu} 3 \mathrm{~d}$ character per unit of L-edge intensity, where the small difference in transition probability reflects the difference in effective nuclear charge of $\mathrm{Fe}^{\mathrm{III}}$ versus $\mathrm{Cu}^{\mathrm{II}}$.

Using the known covalency ${ }^{58}$ and the integrated L-edge intensity of $\left[\mathrm{FeCl}_{4}\right]^{-}$, the integrated intensities of other ferric complexes (Table 1, data column 1) could be used to experimentally determine their covalency averaged over the half- or unoccupied $\mathrm{d}$ orbitals. The metal $\mathrm{d}$ character in the partially occupied antibonding "d" orbitals is found to be $85 \%$ for $\left[\mathrm{FeCl}_{6}\right]^{3-}, 83 \%$ for $\mathrm{Fe}(\mathrm{acac})_{3}, 84 \%$ for $\left[\mathrm{Fe}(\mathrm{ox})_{3}\right]^{3-}$, and $82 \%$

(58) Shadle, S. E.; Hedman, B.; Hodgson, K. O.; Solomon, E. I. J. Am. Chem. Soc. 1995, 117, 2259-2272. 
for $\left[\mathrm{Fe}(\mathrm{ida})_{2}\right]^{-}$indicating a significant ligand $\mathrm{p}$ covalent mixing for each of these high-spin ferric complexes. Similarly, the average covalency over the partially occupied $t_{2}$ and unoccupied e orbitals of the ferric low-spin $\sigma$-donor $\left[\mathrm{Fe}(\operatorname{tacn})_{2}\right]^{3+}$ complex is determined to be $70 \%$ metal d character.

Ligand K-edge XAS can also be used to independently determine the covalency in $\left[\mathrm{FeCl}_{6}\right]^{3-}$. This ferric $O_{h}$ chloride is found to have $17 \%( \pm 1 \%)$ ligand $\mathrm{p}$ character (Figure S4, Supporting Information) and therefore $83 \%$ metal d character. Thus the L-edge methodology is in good agreement with previously established methods for quantitatively determining metal-ligand covalency in model systems.

In addition to covalency, the transition probability is proportional to the number of one-electron XAS transition pathways, which is equivalent to the number of unoccupied one-electron (spin-unrestricted) orbitals in the metal $3 \mathrm{~d}$ manifold. For ferrous systems, the additional electron in the d-manifold must be accounted for when quantitatively determining the covalency of the complex. Thus, while the measured experimental intensity is lower for ferrous complexes as compared to the ferric analogues, the intensity should be renormalized by a factor of $5 / 4$ to account for the loss of one pathway due to the additional electron. Using this methodology, the experimentally determined covalencies of $\left[\mathrm{FeCl}_{4}\right]^{2-},\left[\mathrm{FeCl}_{6}\right],{ }^{4-}$ and $\left[\mathrm{Fe}(\operatorname{tacn})_{2}\right]^{2+}$ are $90 \%$, $92 \%$, and $72 \%$, respectively, as summarized in Table 1, column 2. As with the ferric complexes, the accuracy of the L-edge methodology was further evaluated against ligand K-edge XAS results for the $\left[\mathrm{FeCl}_{4}\right]^{2-}$ complex ${ }^{58}$ that determined $(9 \% \mathrm{Cl} 3 \mathrm{p})$ the metal d component of its wave function to be $90 \%$, in good agreement with the metal L-edge analysis.

Several striking observations emerge from the experimental covalencies in Table 1. First, the metal d character of each of the ferric high-spin complexes is reduced by $\sim 8 \%$ relative to the equivalent ferrous complex. From molecular orbital theory, the deeper binding energy of the $d$ orbitals of the ferric complexes allows greater bonding interactions with the ligand $\mathrm{p}$ orbitals and thus results in increased covalent mixing. The covalent mixing of the low-spin ferric and ferrous complexes, while similar to each other, is dramatically increased relative to the high-spin complexes of the same state. The high-spin ferric complexes have three transitions to the $t_{2}$ orbitals and two transitions to the e levels, whereas the low-spin ferric complex has only one transition to the $t_{2}$ but four to the e set. Since the e orbitals are ligand directed and involved in $\sigma^{*}$ bonding, whereas the $t_{2}$ are involved in $\pi^{*}$ bonding, the increased covalency of the low-spin system reflects the increased contribution to the metal L-pre-edge from the greater mixing of the metal e orbitals with the ligands. For high-spin ferrous complexes, the pre-edge intensity reflects contributions from two transitions to both the $t_{2}$ and e levels. Low-spin ferrous complexes have four transitions to the e level and none to the $\mathrm{t}_{2}$ levels. Again, the $\sigma^{*}$ nature of the e orbitals leads to a higher total covalency in the low-spin ferrous complexes. Finally, the difference in covalency between octahedral and tetrahedral complexes of a given oxidation state is quite small, $85 \%$ versus $81 \%$, respectively, for ferric chlorides, and $92 \%$ versus $90 \%$, respectively, for ferrous chlorides. This will be discussed in section 5 .

4.2. Ligand Field Multiplet Fits of Spectra. Ligand field multiplet simulations of the L-edge spectra for $\left[\mathrm{FeCl}_{6}\right]^{3-}$,

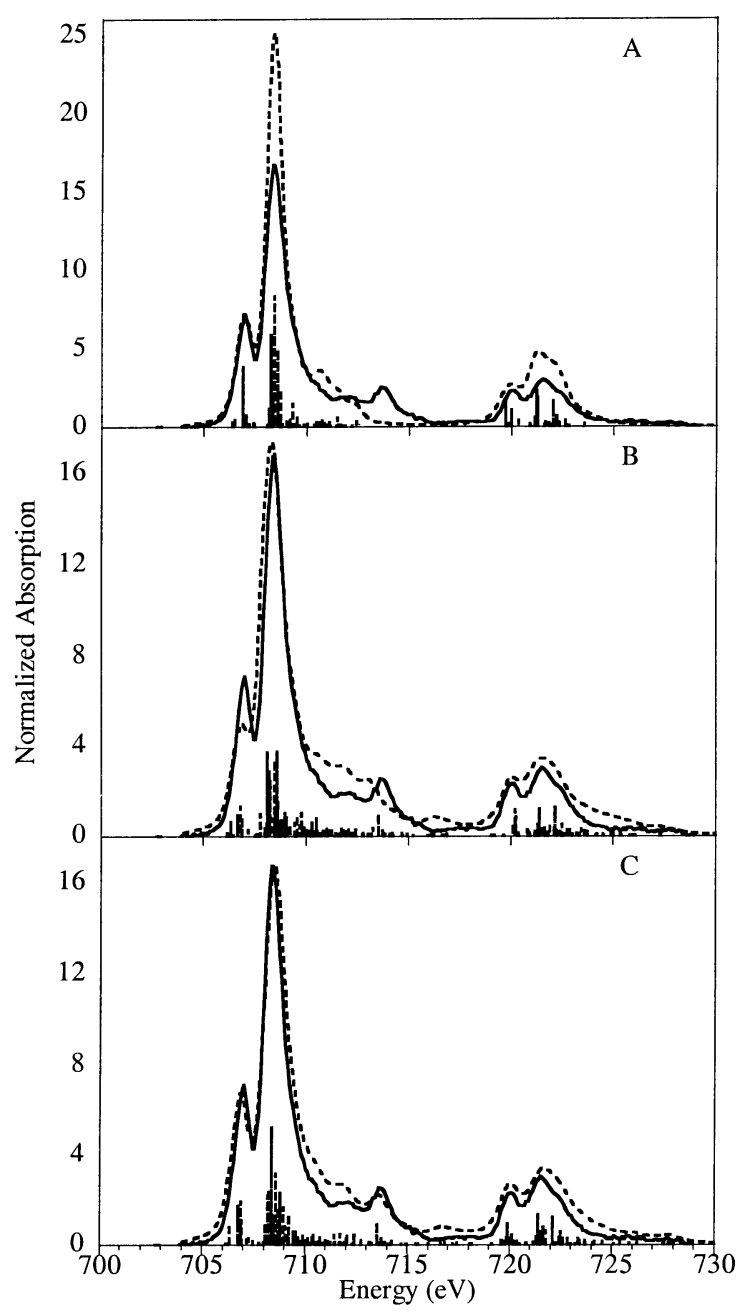

Figure 2. Ligand field multiplet (A), isotropic covalency (B), and DOC (C) simulations of $\left[\mathrm{FeCl}_{6}\right]^{3-}$. Note the difference in simulated intensity in panel A versus panels B and C.

$\left[\mathrm{FeCl}_{6}\right],{ }^{4-}\left[\mathrm{Fe}(\operatorname{tacn})_{2}\right]^{3+}$, and $\left[\mathrm{Fe}(\operatorname{tacn})_{2}\right]^{2+}$ are shown in Figures $2-5$. Spectra were simulated treating the ligand field strength, $10 \mathrm{Dq}$, and the Coulomb repulsion energies, $F_{2}, F_{4}, G_{1}$, and $G_{3}$, as variables. The covalent reduction parameters $\beta$ for the Coulomb repulsion terms are reported for comparison with literature values. The ligand field simulations do not include covalency, except through the reduction of electron-electron repulsion. Therefore, the valence orbitals have pure metal d-orbital character, and the integrated intensity of the simulated spectra correspond to this ionic limit in each case. Furthermore, each simulation was convoluted with a Gaussian function $(\sigma=$ $0.2 \mathrm{eV}$ ) representing instrumental resolution broadening, as well as a Lorentzian function for each of the $\mathrm{L}_{3}$ - and the $\mathrm{L}_{2}$-edges ( $\Gamma_{1}=0.2 \mathrm{eV}$ and $\Gamma_{2}=0.4 \mathrm{eV}$, respectively) representing the core-hole lifetime, which is different for each pre-edge.

The spectrum of $\left[\mathrm{FeCl}_{6}\right]^{3-}$ was best simulated with a $10 D q=1.2 \mathrm{eV}$ and $\beta=55 \%$, in reasonable agreement with optical spectroscopy $(10 D q=1.4 \mathrm{eV}, \beta=55 \%) .{ }^{59}$ The best ligand field multiplet simulation of $\left[\mathrm{FeCl}_{6}\right]^{3-}$ qualitatively reflects the main features of the L-edge spectrum reasonably well (Figure 2A). The main $\mathrm{L}_{3}$-edge shape is reproduced, including a lower-energy peak at $707 \mathrm{eV}$ and the $\mathrm{L}_{2}$-edge clearly consists of two absorption envelopes. However, the simulation

(59) Yamatera, H.; Kato, A. Bull. Chem. Soc. Jpn. 1968, 41, 2220. 


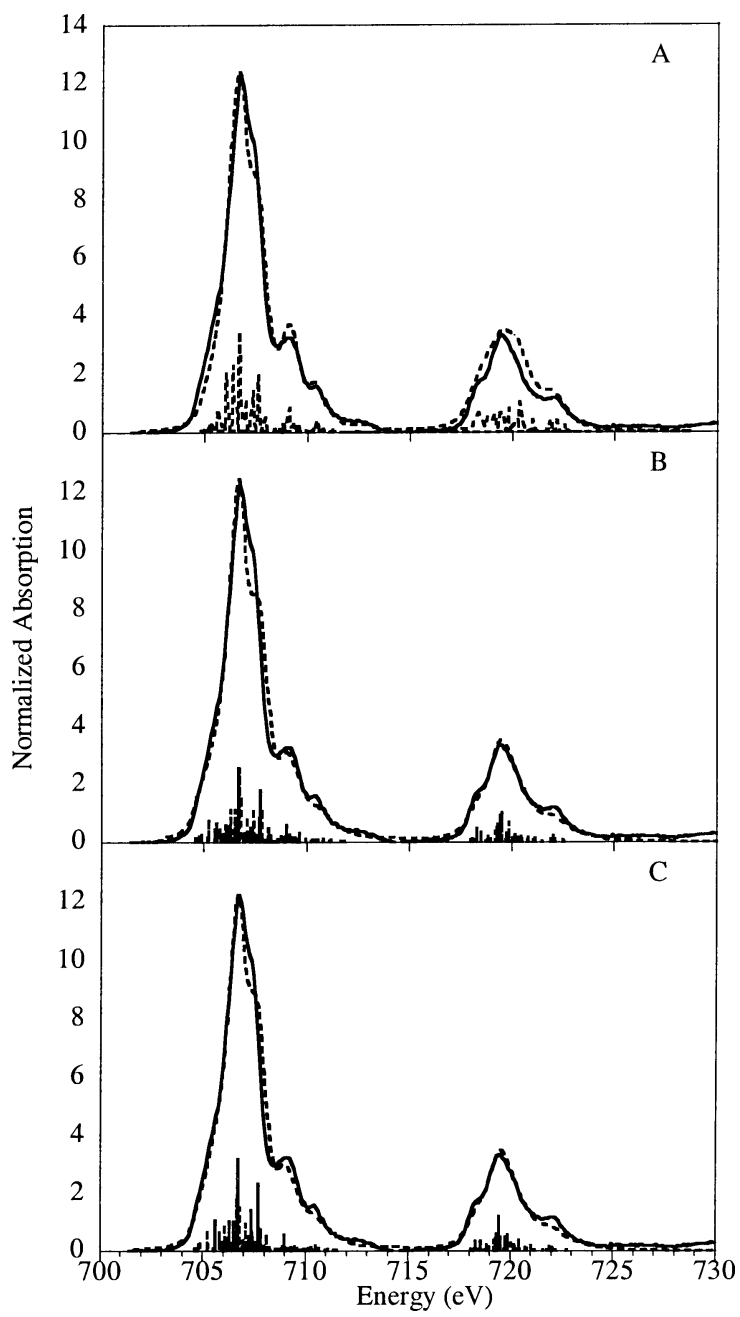

Figure 3. Ligand field multiplet (A), isotropic covalency (B), and DOC (C) simulations of $\left[\mathrm{FeCl}_{6}\right]^{4-}$.

fails to reproduce the intensity of the CT region $(712-716 \mathrm{eV})$ of the spectrum and significantly overestimates the main $\mathrm{L}_{3}$ (708-712) and $\mathrm{L}_{2}$ intensity $(719-725 \mathrm{eV})$.

The best simulation of the ferric tetrahedral complex $\left[\mathrm{FeCl}_{4}\right]^{-}$ was obtained (Figure S1A) using parameters of $10 \mathrm{Dq}=$ $-0.7 \mathrm{eV}$ and $\beta=60 \%$. This is in reasonable agreement with UV-vis absorption data, which found $10 D q=-0.81 \mathrm{eV}$ and $\beta=40 \% ; 60$ however, the simulation is clearly not an adequate fit to the data. Again, the CT region of the spectrum (716-720 $\mathrm{eV}$ ) is not well reproduced by the ligand field model, and the $\mathrm{L}_{3^{-}}$and $\mathrm{L}_{2}$-edge intensity are overestimated.

The best ligand field multiplet simulation of $\left[\mathrm{FeCl}_{6}\right]^{4-}$ gave $\beta=86 \%$ and $10 D q=0.6 \mathrm{eV}$ and reproduces the spectrum extremely well (Figure 3A). The energy of the main absorption peak and three higher-energy shoulders of the $\mathrm{L}_{3}$-edge as well as the primary peak and high-energy shoulder of the $\mathrm{L}_{2}$-edge are well fit. While the low-energy shoulder of the $\mathrm{L}_{2}$-edge is somewhat broadened relative to the data, it has been shown that individual multiplet lines are broadened to varying degrees, ${ }^{61}$ and this may be the cause of the small discrepancy in the fit. Unlike the $O_{h}$ ferric case, the intensities of the simulated $\mathrm{L}_{3^{-}}$and $\mathrm{L}_{2}$-edges correctly reproduce the data.

(60) Deaton, J. C.; Gebhard, M. S.; Solomon, E. I. J. Am. Chem. Soc. 1989, 28 , $877-889$.

(61) Okada, K.; Kotani, A.; Ogasawara, H.; Seino, Y.; Thole, B. T. Phys. Rev. B. 1993, 47, 6203-6206.

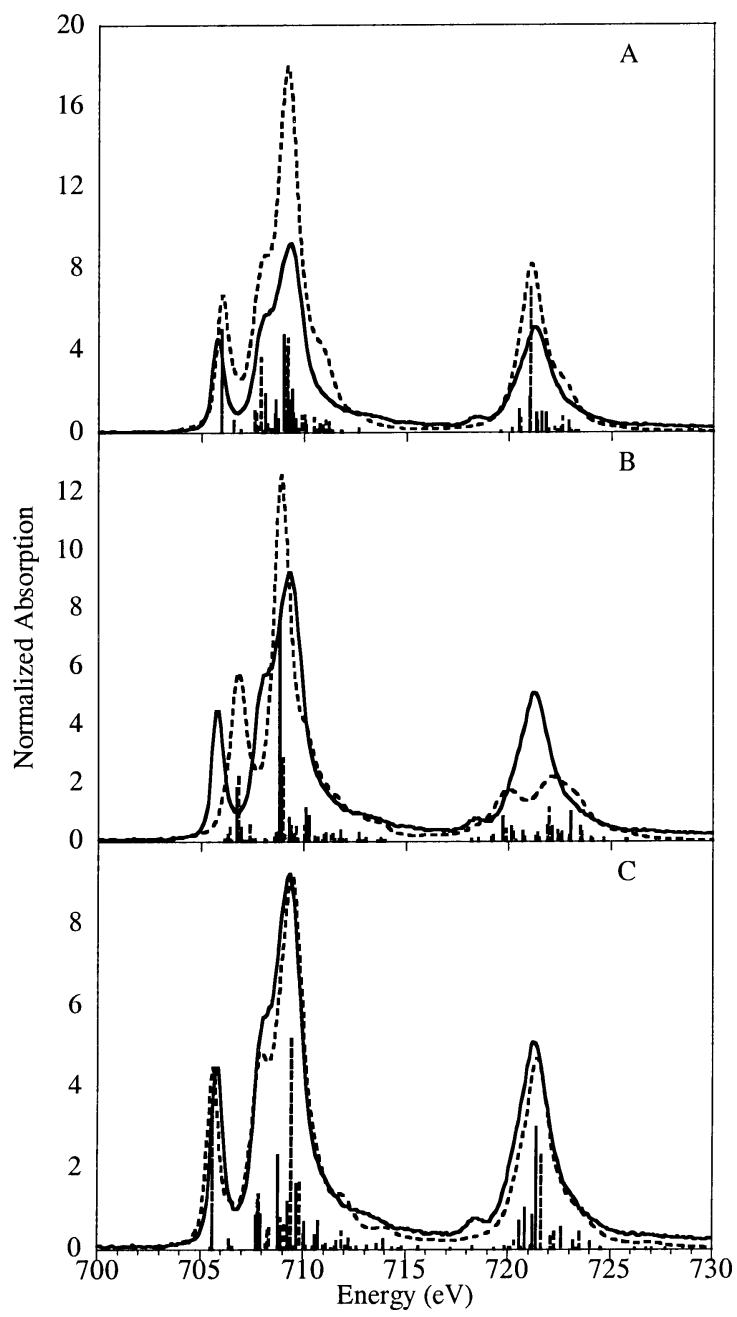

Figure 4. Ligand field multiplet (A), isotropic covalency (B), and DOC (C) simulations of $\left[\mathrm{Fe}(\operatorname{tacn})_{2}\right]^{3+}$. Note the difference in simulated intensity in each panel.

The best simulation of the ferrous tetrahedral complex $\left[\mathrm{FeCl}_{4}\right]^{2-}$ is shown in Figure S2A with parameters of $10 \mathrm{Dq}=$ $-0.4 \mathrm{eV}$ and $\beta=85 \%$, in reasonable agreement with $\mathrm{UV}-$ vis absorption data, which found $10 D q=-0.51 \mathrm{eV}$ and $\beta=87 \% .{ }^{62}$ Overall, the spectrum is quite well reproduced by the simulation. Energy splittings and most intensity envelopes are mimicked very well, with only a slight overestimation of the intensity in the $\mathrm{L}_{3}$-edge at $712 \mathrm{eV}$ and in the $\mathrm{L}_{2}$-edge between 721 and $723 \mathrm{eV}$.

The simulation of the low-spin $\left[\mathrm{Fe}(\operatorname{tacn})_{2}\right]^{3+}$ complex (Figure 4A) with $10 D q=2.50 \mathrm{eV}$ and $\beta=65 \%$ is in good agreement with optical data $(10 D q=2.6 \mathrm{eV}$ and $\beta=59 \%){ }^{33,63}$ In general, the energy splittings are found to be in reasonable agreement between the multiplet simulation and the data (Figure 4A). However, the simulation significantly overestimates the intensity of all of the $\mathrm{L}_{3}$ and $\mathrm{L}_{2}$ pre-edge features. Notably, the closest match between the simulated spectrum and the data occurs in the feature at $706 \mathrm{eV}$, attributed to the lone half-occupied $\mathrm{t}_{2 \mathrm{~g}}$ orbital in the $\left(\mathrm{t}_{2 \mathrm{~g}}\right)^{5}\left(\mathrm{e}_{\mathrm{g}}\right)^{0}$ ferric ground state ion. The difference in overestimation of intensity in the simulation between the feature at $706 \mathrm{eV}$ and the multiplets at $707-714 \mathrm{eV}$ is indicative

(62) Furlani, C.; Cervone, E.; Valenti, V. J. Inorg. Nuc. Chem. 1963, 25, 159163.

(63) Wieghardt, K.; Küppers, H.-J.; Weiss, J. Inorg. Chem. 1985, 24, 30673071 . 


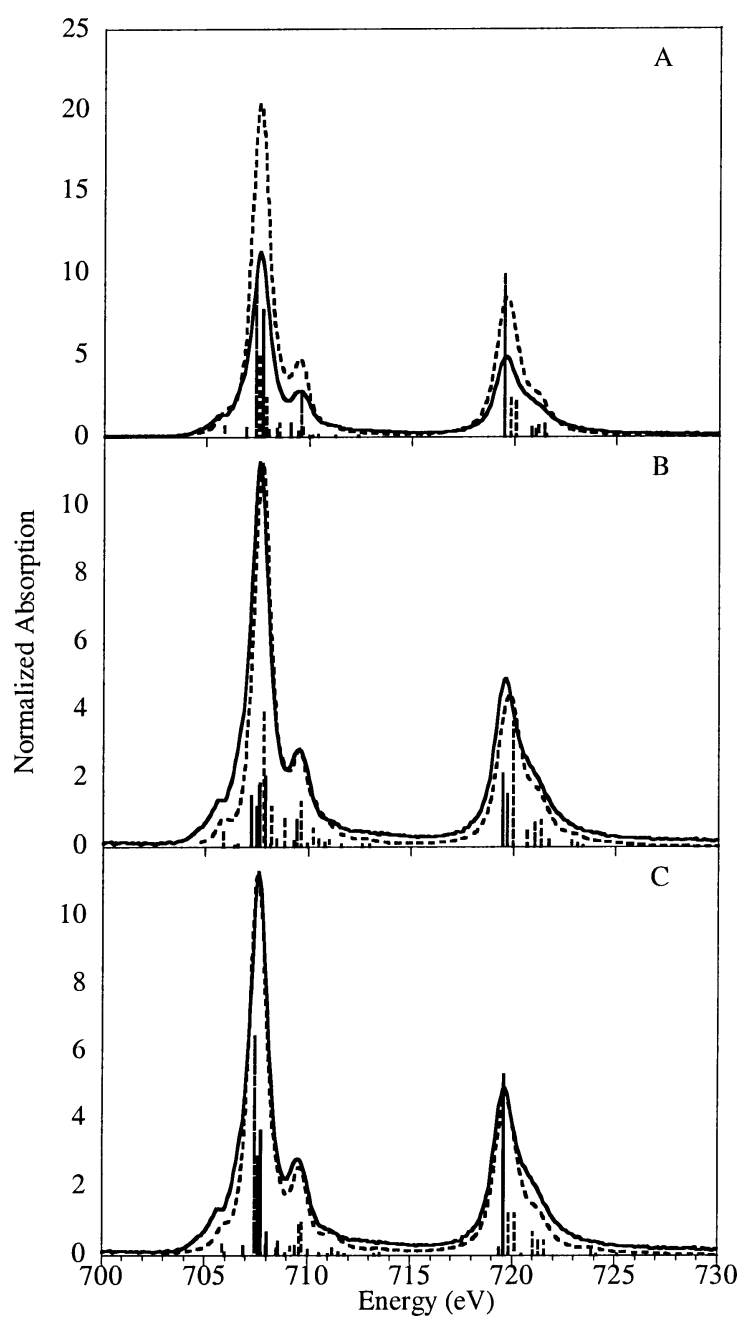

Figure 5. Ligand field multiplet (A), isotropic covalency (B), and DOC (C) simulations of $\left[\mathrm{Fe}(\operatorname{tacn})_{2}\right]^{2+}$. Note the difference in simulated intensity in panel A versus panels B and C.

of the covalency differences of the individual $d$ orbitals that contribute to the multiplets, and the mismatch in simulated intensity to the data indicates the inadequacy of the ligand field multiplet method to model the ground state wave function of $\left[\mathrm{Fe}(\operatorname{tacn})_{2}\right]^{3+}$.

As shown in Figure $5 \mathrm{~A}$, the data of $\left[\mathrm{Fe}(\operatorname{tacn})_{2}\right]^{2+}$ can be reasonably simulated using the ligand field multiplet method with parameters $10 \mathrm{Dq}=2.1 \mathrm{eV}$ and $\beta=80 \%$. Analysis of UV-vis absorption data ${ }^{33,63}$ found $10 D q=2.35 \mathrm{eV}$ which compares well with the value of $2.1 \mathrm{eV}$ found here. However, the $\beta$ value of $80 \%$ is somewhat higher than that determined from UV-vis absorption $(\beta=61 \%)$. The energy splittings of the simulated features reproduce the experimental spectrum very well. The simulated spectrum does not correctly estimate the experimental intensity, however.

In general, the ligand field multiplet simulations reproduce the multiplet pattern of the experimental spectra for the ferrous complexes reasonably well. In the case of the high-spin $\mathrm{FeCl}_{6}{ }^{4-}$ complex, the experimental total intensity is also well fit by the ligand field simulation. For the low-spin $\left[\mathrm{Fe}(\operatorname{tacn})_{2}\right]^{2+}$ complex, however, the intensity is significantly overestimated by the calculated spectrum. From section 1, the covalency of the ferrous site is quite low for the high-spin complex (92\% d orbital character) but is relatively high ( $72 \%$ d orbital character) in the case of the low-spin ferrous complex. Thus, the reduction in the Slater integrals and the resulting compression of the multiplet spectrum successfully model the high-spin ferrous data but do not successfully reproduce the low-spin $\left[\mathrm{Fe}(\operatorname{tacn})_{2}\right]^{2+}$. Moreover, the model breaks down for both low- and high-spin ferric complexes. In addition to the significantly overestimated total intensity in both pre-edges of the simulated spectra for the highspin $\left[\mathrm{FeCl}_{4}\right]^{-}$and $\left[\mathrm{FeCl}_{6}\right]^{3-}$ complexes, the $\mathrm{CT}$ satellite region of the calculated spectrum lacks intensity. For the low-spin $\left[\mathrm{Fe}(\operatorname{tacn})_{2}\right]^{3+}$ complex, while the energies of the multiplets are reasonable, the total intensity for each L-edge is significantly overestimated, the overestimation is not constant throughout a given edge, and the intensities are not reproduced in the CT region. From the total intensities in Table 1, the ferric complexes are more covalent than their ferrous counterparts. In these cases where the covalency is high and where CT satellite bands are observed, and in the case of low-spin ferric complexes where the covalencies of the $t_{2 g}$ and $e_{g}$ orbitals are expected to be quite dissimilar, the inclusion of separate $t_{2 g}$ and $e_{g}$ covalency effects into the multiplet calculation is necessary.

4.3. Covalency Fits of Spectra. In the ligand field multiplet model, the ground state is approximated by a single electronic configuration $\mathrm{d}^{n}{ }^{64}$ Including charge transfer allows for mixing of the metal valence $d$ orbitals with ligand valence $\mathrm{p}$ orbitals and therefore necessitates multiple configurations. The charge transfer multiplet model adds a $\mathrm{d}^{n+1} L$ configuration to the single configuration $\mathrm{d}^{n}$ ground state of the ligand field model. The lowest energy $\mathrm{d}^{n+1} \underline{L}$ configuration is set at an energy $\Delta_{\mathrm{GS}}$ above the lowest energy $\mathrm{d}^{n}$ configuration, and the two states are coupled by configuration interaction, represented by the covalent mixing term $T$,

$$
T=\left\langle 3 d^{n}|h| 3 d^{n+1} \underline{L}\right\rangle
$$

where $h$ is the molecular Hamiltonian and $T$ is proportional to metal-ligand overlap. In the case of a two-state system, the ground state interaction matrix and its solutions are

$$
\begin{gathered}
H=\left|\begin{array}{ll}
0 & T \\
T & \Delta_{\mathrm{GS}}
\end{array}\right| \\
\Psi_{\mathrm{GS}, B}=\alpha_{1}\left|3 d^{n}\right\rangle+\beta_{1}\left|3 d^{n+1} \underline{L}\right\rangle \\
\Psi_{\mathrm{GS}, A B}=\beta_{1}\left|3 d^{n}\right\rangle-\alpha_{1}\left|3 d^{n+1} \underline{L}\right\rangle
\end{gathered}
$$

Similarly, the bonding $\left(\Psi_{\mathrm{ES}, B}\right)$ and antibonding $\left(\Psi_{\mathrm{ES}, A B}\right)$ charge transfer solutions for the XAS excited states may be written as

$$
\begin{gathered}
\Psi_{\mathrm{ES}, B}=\alpha_{2}\left|\underline{c} 3 d^{n+1}\right\rangle+\beta_{2}\left|\underline{c} 3 d^{n+2} \underline{L}\right\rangle \\
\Psi_{\mathrm{ES}, A B}=\beta_{2}\left|\underline{c} 3 d^{n+1}\right\rangle-\alpha_{2}\left|\underline{c} 3 d^{n+2} \underline{L}\right\rangle
\end{gathered}
$$

where the charge transfer configuration in the excited state is at an energy $\Delta^{\prime}\left(=\Delta_{\mathrm{GS}}+U-Q\right.$, where $U$ is the $3 \mathrm{~d}-3 \mathrm{~d}$ electron correlation and $Q$ is the $2 \mathrm{p}-3 \mathrm{~d}$ correlation) above the ground configuration, and the interaction parameters $(T)$ are approximated as equivalent in the ground and excited states. ${ }^{64}$ The resulting L-edge spectrum consists of a main absorption feature followed by a small satellite feature to higher energy.

In the case of a $\mathrm{d}^{n}$ system, where multiple electronic states can contribute to the ground state of the system, multiple satellite

(64) de Groot, F. M. F. Chem. Rev. 2001, 101, 1779-1808. 
features are possible, modifying the multiplet spectrum beyond the compression of multiplets found by reducing the Slater integrals as in section 1 . The additional electronic states within the $\mathrm{d}^{n+1} \underline{L}$ configuration reduce the effective $\Delta$ for the higher lying states and thus compress the multiplet spectrum. ${ }^{64}$ Furthermore, the addition of the $\mathrm{d}^{n+1} L$ configuration in the ground state and the $c \mathrm{~d}^{n+2} L$ configuration in the excited state produces additional shake-up intensity to the high-energy side of the $\mathrm{L}_{3}$ - and $\mathrm{L}_{2}$-edges. Using charge transfer multiplet theory, simulations of the spectra have been obtained that explicitly include isotropic covalency where the interaction terms between the metal and the ligands are equal for all $\mathrm{d}$ orbitals.

4.3.1. Isotropic Covalency. In the case of $\left[\mathrm{FeCl}_{6}\right]^{3-}$, the charge transfer multiplet calculation with isotropic covalency $\left(\Delta_{\mathrm{GS}}=0.1 \mathrm{eV}\right.$ and $\left.T=1.15 \mathrm{eV}\right)$ greatly modifies the simulated spectrum (Figure 2B). There is a reduction in total intensity due to covalency, and significant intensity has been redistributed to higher energy (713 to $717 \mathrm{eV}$ ) relative to the ligand field simulation in Figure 2A. However, the intensity of the lowenergy $\mathrm{L}_{3}$ peak $(707 \mathrm{eV})$ is underestimated and not well fit. The $\mathrm{L}_{2}$-edge is well simulated with two features correctly separated in energy and with the correct intensity distribution.

The isotropic covalency simulation of the ferrous octahedral high-spin $\left[\mathrm{FeCl}_{6}\right]^{4-}$ complex faithfully reproduces the data with parameters of $\Delta_{\mathrm{GS}}=3.0 \mathrm{eV}$ and $T=0.6 \mathrm{eV}$ (Figure 3B). The L-edge absorption features are well modeled in energy and intensity. However, the simulation is not greatly improved relative to the ligand field multiplet fit without charge transfer included (Figure 3A); the total intensity is only slightly reduced, and little intensity is redistributed to higher energy. This is consistent with a ferrous ion that is mainly ionic in character as found in section 1 .

Using the charge-transfer model with isotropic covalency to simulate the data of low-spin $\left[\mathrm{Fe}(\operatorname{tacn})_{2}\right]^{3+}$ results in a larger intensity redistribution of intensity (Figure 4B). The data were simulated with $\Delta_{\mathrm{GS}}=2.8 \mathrm{eV}$ and $T=1.4 \mathrm{eV}$, which correctly reproduce the total covalency of the complex as found by integrating the multiplet intensity (vide supra). However, the simulated intensity in the $\mathrm{L}_{2}$-edge is $\sim 20 \%$ of that for the simulated $\mathrm{L}_{3}$-edge, whereas the experimental $\mathrm{L}_{2}$-edge is $\sim 50 \%$ of that for the $\mathrm{L}_{3}$-edge, indicating a severe breakdown in the isotropic covalency simulation. ${ }^{65,66}$ Thus, a model including only isotropic covalency is not sufficient to fit these data.

An isotropic covalency charge transfer simulation with $\Delta_{\mathrm{GS}}=2.8 \mathrm{eV}$ and $T=1.1 \mathrm{eV}$ reproduces the data of low-spin $\left[\mathrm{Fe}(\operatorname{tacn})_{2}\right]^{2+}$ very well (Figure $5 \mathrm{~B}$ ). The main $\mathrm{L}_{3}$-edge peaks at 706 and $710 \mathrm{eV}$ are correctly spaced in energy, and the intensity ratio matches the data. The main $\mathrm{L}_{2}$-edge feature and high-energy shoulder are also reproduced. The main difference between the ligand field multiplet and isotropic covalency simulation for $\left[\mathrm{Fe}(\operatorname{tacn})_{2}\right]^{2+}$ is the significant reduction in total simulated intensity resulting from the inclusion of averaged covalency.

Thus, while the ferrous L-edges are well fit by a model that simply allows for a small reduction in total intensity through isotropic covalency, ferric complexes are not. The simulation of the high-spin ferric complex in Figure 2B fails to reproduce

(65) Thole, B. T.; van der Laan, G. Phys. Rev. B. 1988, 38, 3158-3171.

(66) The $L_{3} /\left(L_{3}+L_{2}\right)$ intensity, known as the branching ratio, is indicative of the spin-state of the metal ion. See ref 65
Table 2. Parameters Used in DOC Simulations

\begin{tabular}{lcrccr}
\hline & $\Delta_{G S}$ & \multicolumn{1}{c}{$\Delta^{\prime a}$} & $T_{\mathrm{e}}$ & \multicolumn{1}{c}{$T_{\mathrm{t}}$} & $10 D q_{\text {ion }}$ \\
\hline$\left[\mathrm{FeCl}_{4}\right]^{-}$ & 0.10 & -2.40 & 1.10 & 1.50 & -0.50 \\
{$\left[\mathrm{FeCl}_{6}\right]^{3-}$} & 0.10 & -2.20 & 1.75 & 0.90 & 1.20 \\
{$\left[\mathrm{FeCl}_{4}\right]^{2-}$} & 3.00 & 2.00 & 1.45 & 1.45 & -0.30 \\
{$\left[\mathrm{FeCl}_{6}\right]^{4-}$} & 2.25 & 1.75 & 0.90 & 0.45 & 0.60 \\
$\mathrm{Fe}(\mathrm{acac})_{3}$ & 0.80 & -1.50 & 1.70 & 0.90 & 1.50 \\
{$\left[\mathrm{Fe}(\mathrm{ox})_{3}\right]^{3-}$} & 0.80 & -1.50 & 1.60 & 0.90 & 1.50 \\
{$\left[\mathrm{Fe}(\mathrm{ida})_{2}\right]^{-}$} & 0.60 & -1.40 & 1.70 & 0.90 & 1.60 \\
{$\left[\mathrm{Fe}(\mathrm{tacn})_{2}\right]^{3+}$} & 2.80 & 0.80 & 3.40 & 0.90 & 2.20 \\
{$\left[\mathrm{Fe}(\mathrm{tacn})_{2}\right]^{2+}$} & 2.80 & 2.10 & 1.80 & 0.30 & 1.70 \\
\hline
\end{tabular}

${ }^{a} \Delta^{\prime}=\Delta_{\mathrm{GS}}+\mathrm{U}-\mathrm{Q}$

the multiplet pattern of the $\mathrm{L}_{3}$-edge and while additional intensity has been shifted into the shake-up CT region, the energies of these features are not reproduced. For the low-spin complex (Figure 4B), the isotropic covalency simulation does not predict the correct ground state and thus does not reproduce the experimental data whatsoever.

4.3.2. Differential Orbital Covalency. In the above simulations, a single interaction parameter $T$ was used to account for LMCT. This is not consistent with the actual bonding situation in $O_{h}$ and $T_{d}$ complexes, however. The cubic ligand field splits the d-manifold into the $t_{2}$ and e symmetry blocks, separated in energy, having different metal-ligand $\sigma$ and $\pi$ overlap characteristics, and thus having different covalencies. To model the differential orbital covalency of the different symmetry branches of $d$ orbitals, the charge transfer model was expanded to include an interaction parameter for each d-orbital symmetry, $T\left(\mathrm{t}_{2}\right)$ and $T(\mathrm{e})$. For octahedral complexes, the $T(\mathrm{e})$ term is greater than $T\left(\mathrm{t}_{2}\right)$, and vice versa for tetrahedral complexes, in accordance with the differences in the $\sigma$ and $\pi$ bonding exhibited in $O_{h}$ and $T_{d}$ ligand fields. It should be noted that the in-plane (IP) and out-of-plane (OP) orbitals of the free ligand (before interaction with the metal) might in some cases be split in energy, leading to the necessity for two $\Delta$ values in the calculations, $\Delta_{\mathrm{t} 2 \mathrm{~g}}$ and $\Delta_{\text {eg }}$. However, in the case of the chloride complexes, the pi and sigma ligand states are at equal energy before CI, whereas for the amine complexes, the ligand pi orbitals do not interact with the metal d orbitals. For the oxygen based ligand systems, DFT calculations give the "pi" and "sigma" ligand orbital energy separation at less than $0.6 \mathrm{eV}$ before binding to the metal ion. Using this energy separation, approximating the ligand pi and sigma orbitals with a single delta leads to an error of $\sim 0.25 \%$ metal $\mathrm{d}$ character in each orbital set and an overestimation of the DOC by $\sim 0.5 \%$. Thus, for the molecules in this study, the use of two deltas (one for the $\sigma$ and one for the $\pi$ symmetry free ligand orbitals) is not necessary.

The charge-transfer multiplet simulation of $\left[\mathrm{FeCl}_{6}\right]^{3-}$ including DOC (parameters in Table 2) reproduces the data extremely well as shown in Figure 2C. Both features of the main $\mathrm{L}_{3}$-edge (707 and $709 \mathrm{eV}$ ) are reproduced in energy and intensity, and the experimental $\mathrm{L}_{3}$ shoulder features $(711-716 \mathrm{eV})$ are present in the simulation. The DOC calculation also fits the feature at $714 \mathrm{eV}$ that was not well fit in either the ligand field or isotropic covalency simulations. The simulated $\mathrm{L}_{2}$-edge has two features separated in energy by $\sim 2 \mathrm{eV}$ and correctly mimics the intensity ratio of the experimental data, unlike the ligand field multiplet simulation. Simulations of the other ferric high-spin species listed in Table 1 are found in the Supporting Information. 
The DOC simulation for the ferrous high-spin $\left[\mathrm{FeCl}_{6}\right]^{4-}$ complex is shown in Figure $3 \mathrm{C}$. The main $\mathrm{L}_{3}$ and $\mathrm{L}_{2}$ multiplets are reproduced quite well, as are the shoulders to the high-energy side of both edges (708 and $722 \mathrm{eV}$, respectively). There is little difference between the DOC fit and the isotropic covalency fit (Figure 3B) or ligand field multiplet fit (Figure 3A), however. Thus, while the DOC methodology may be used to simulate the spectrum, it is not necessary.

The charge-transfer multiplet simulation of low-spin [Fe$\left.(\operatorname{tacn})_{2}\right]^{3+}$ excellently reproduces the experimental data (Figure 4C). The $\mathrm{L}_{3}$-edge consists of two absorption envelopes at 705706 and $707-717 \mathrm{eV}$ that involves transitions to the $\mathrm{t}_{2 \mathrm{~g}}{ }^{6} \mathrm{e}_{\mathrm{g}}{ }^{0}$ and $\mathrm{t}_{2 \mathrm{~g}}{ }^{5} \mathrm{e}_{\mathrm{g}}{ }^{1}$ final state multiplets, respectively. The calculated spectrum correctly models the intensity ratio and energy separation of these multiplet envelopes, including the small shoulder at $712 \mathrm{eV}$. The $\mathrm{L}_{2}$-edge data consist of a small feature at $718 \mathrm{eV}$ $\left(\mathrm{t}_{2 \mathrm{~g}}{ }^{6} \mathrm{e}_{\mathrm{g}}{ }^{0}\right.$ multiplet), as well as a much larger feature centered at $722 \mathrm{eV}$ with a poorly resolved, small shoulder at $\sim 723 \mathrm{eV}$ $\left(\mathrm{t}_{2 \mathrm{~g}}{ }^{5} \mathrm{e}_{\mathrm{g}}{ }^{1}\right.$ multiplets). While the calculated intensity is too low relative to experiment at $718 \mathrm{eV}$, a transition is present at the correct energy in the simulation. The low intensity in this feature may be due to a lifetime broadening used in the simulation that is too large for the individual transition. ${ }^{61}$ Furthermore, the two features at $720-725 \mathrm{eV}$ account for the majority of the intensity in the edge and are very well fit by the simulation.

Similarly, the charge transfer simulation for the ferrous lowspin $\left[\mathrm{Fe}(\operatorname{tacn})_{2}\right]^{2+}$ complex (Figure 5C) reproduces the experimental data well for the main features and shoulders of both the $\mathrm{L}_{3}$ and $\mathrm{L}_{2}$ multiplets. The primary difference in the simulation with the inclusion of DOC is a reduction in simulated intensity relative to the LFM simulation, consistent with the reduction in metal d orbital character. In the multiplet splittings, only a slight difference can be observed between the ligand field and charge transfer multiplet fits to the data (Figure 5A and C, respectively). A very small shoulder to the high-energy side of the $\mathrm{L}_{3}$ multiplets $(\sim 711 \mathrm{eV})$ is found, which is fit marginally better with the inclusion of either isotropic or differential orbital covalency.

4.3.2.1. Extraction of Covalency. The ground state parameters found from applying the charge transfer multiplet methodology with DOC, listed in Table 2, were used to determine the covalency of the half- or unoccupied metal $t_{2}$ and e orbitals in the ground state. The ground state wave function in eq 4.1 is a sum of weak-field multiplet terms deriving from the $3 \mathrm{~d}^{n}$ and $3 \mathrm{~d}^{n+1} \underline{L}$ configurations. Rewritten for covalency considerations, the ground state can alternatively be written as a sum of strongfield components for covalency considerations.

$$
\begin{gathered}
\Psi_{\mathrm{GS}}=\alpha_{1}\left|t_{2 g}^{n} e_{g}^{0}\right\rangle+\alpha_{2}\left|t_{2 g}^{n-1} e_{g}^{1}\right\rangle+\alpha_{3}\left|t_{2 g}^{n-2} e_{g}^{2}\right\rangle+\alpha_{4}\left|t_{2 g}^{n-3} e_{g}^{3}\right\rangle+ \\
\alpha_{5}\left|t_{2 g}^{n-4} e_{g}^{4}\right\rangle+\alpha_{6}\left|t_{2 g}^{n+1} e_{g}^{0} \underline{L}\right\rangle+\alpha_{7}\left|t_{2 g}^{n} e_{g}^{1} \underline{L}\right\rangle+\alpha_{8}\left|t_{2 g}^{n-1} e_{g}^{2} \underline{L}\right\rangle+ \\
\alpha_{9}\left|t_{2 g}^{n-2} e_{g}^{3} \underline{L}\right\rangle+\alpha_{10}\left|t_{2 g}^{n-3} e_{g}^{4} \underline{L}\right\rangle \\
\sum_{i}\left(\alpha_{i}^{2}\right)=1
\end{gathered}
$$

This ground state $\left(\Psi_{\mathrm{GS}}\right)$ is comprised of a linear combination of contributions from the main and CT $\mathrm{t}_{2 \mathrm{~g}}{ }^{n} \mathrm{e}_{\mathrm{g}}{ }^{m}$ configurations. The latter reflects the covalency, which can be obtained by projection onto a hypothetical state $\left(\Psi_{\mathrm{H}}\right)$ in which all multiplet effects have been removed $\left(H_{\mathrm{ee}}=H_{\mathrm{LS}}=0\right)$, leaving only the ligand field splitting $\left(H_{\mathrm{LF}}\right)$ to contribute to multiplet splitting.
The Hamiltonian for this hypothetical state $\left(H_{\mathrm{H}}\right)$ can be written in the form

$$
H_{\mathrm{H}}=\left\langle 3 d^{n}+3 d^{n+1} \underline{L}\left|H_{\mathrm{ave}}+H_{\mathrm{LF}}\right| 3 d^{n}+3 d^{n+1} \underline{L}\right\rangle
$$

The eigenfunctions corresponding to this Hamiltonian, $\Psi_{\mathrm{H}}^{i}$, are the strong field configurations listed in eq 4.2 for $\Psi_{\mathrm{GS}}$ such that

$$
\left\langle\Psi_{\mathrm{GS}} \mid \Psi_{\mathrm{H}}^{i}\right\rangle=\alpha_{i}
$$

Thus, projecting the hypothetical state onto the ground state gives the coefficients of the strong field wave function components, $\alpha_{i}$, in the ground state. The covalency of the individual $t_{2 g}$ or $e_{g}$ orbitals is found from the $\alpha_{i}{ }^{2}$ of the $t_{2 g}{ }^{n+1} e_{g}{ }^{m}$ and $\mathrm{t}_{2 \mathrm{~g}}{ }^{n} \mathrm{e}_{\mathrm{g}}{ }^{m+1}$ configurations corresponding to LMCT from the $\mathrm{t}_{2 \mathrm{~g}}{ }^{n} \mathrm{e}_{\mathrm{g}}{ }^{m}$ ground state into the metal $\mathrm{t}_{2 \mathrm{~g}}$ and $\mathrm{e}_{\mathrm{g}}$ orbitals, respectively.

In the projection methodology, the $3 \mathrm{~d}^{n}+3 \mathrm{~d}^{n+1} \underline{L}$ ground state multiplet, comprised of a linear combination of contributions from one or more $\mathrm{t}_{2 \mathrm{~g}}{ }^{x} \mathrm{eg}_{\mathrm{g}}^{y}$ configurations, is mapped into the individual strong field configurations. This projection is accomplished within the program suite by linking the ground and hypothetical states to a $4 \mathrm{~s}$ to $4 \mathrm{p}$ transition. Any multiplet effects from the $4 \mathrm{~s}$ and $4 \mathrm{p}$ contributions to the wave function are turned off; therefore the energies of the configurations are completely determined by the orbital occupancies of the ligand field split configurations, $10 D q$, and by the metal to free-ligand energy separation, $\Delta$.

The intensity of this transition is distributed among the strong field $3 \mathrm{~d}^{n}$ and $3 \mathrm{~d}^{n+1}$ configurations according to their contributions to the ground-state wave function. For a high-spin octahedral ferric complex, the primary intensity is in the $\mathrm{t}^{3} \mathrm{e}^{2}$ ground-state configuration. The intensities corresponding to the $\mathrm{t}^{4} \mathrm{e}^{2}$ and $\mathrm{t}^{3} \mathrm{e}^{3} \mathrm{~d}^{n+1} L$ contributions are at energies of $E_{\text {ave }}-4 D q_{\mathrm{H}}$ and $E_{\text {ave }}+6 D q_{\mathrm{H}}$, respectively. The covalency for an individual $\mathrm{d}^{n+1}$ state is simply the calculated intensity of that state renormalized to include the number of one-electron pathways contributing to the intensity. Of the five one-electron transitions from the ground state configuration to the charge transfer $\mathrm{d}^{n+1} \underline{L}$ configuration, only two transitions correspond to intensity into the e orbitals, whereas three of five correspond to transitions to the $t_{2}$ orbitals. The intensity of the $t^{3} \mathrm{e}^{2} \rightarrow \mathrm{t}^{3} \mathrm{e}^{3} L$ transition at $E_{\text {ave }}+6 D q_{\mathrm{H}}$, for example, represents the summed contribution of the LMCT to the two half-occupied orbitals of e symmetry. Thus the renormalized intensity in the half-occupied e orbitals is $I^{\prime}=I\left(E_{\text {ave }}+6 D q_{\mathrm{H}}\right)^{* 5 / 2}$, whereas the renormalized intensity in the half-occupied $t_{2}$ orbitals is $I^{\prime}=I\left(E_{\text {ave }}-4 D q_{\mathrm{H}}\right)^{* 5 / 3}$. In a similar manner, for a low-spin ferric complex, the renormalized intensity in the unoccupied e orbitals $I^{\prime}=I\left(E_{\text {ave }}-14 D q_{\mathrm{H}}\right)^{* 5 / 4}$, whereas the renormalized intensity in the single half-occupied $\mathrm{t}_{2}$ orbital $I^{\prime}=I\left(E_{\text {ave }}-24 D q_{\mathrm{H}}\right) * 5 / 1$.

For the compounds in Table 1, the total covalency determined by averaging the $t_{2}$ and e symmetry orbitals from the projection method was 1-13\% lower than that found experimentally by integrating the intensity over the multiplets as described in section 1. Integrating the experimental L-edge intensity is a more direct method for determining the total covalency of a system than relying upon the spectral simulation. Thus, the DOC found through the projection method was scaled to the value from the total experimental L-edge intensity, which changes the DOC 
value by at most $2 \%$, which is within the error of the technique. A complete listing of individual orbital covalencies and DOC (scaled to the total intensity) found for the L-edge data is given in Table 1.

Spin-unrestricted molecular orbital calculations (DFT, BP86) were performed for the model complexes studied here for comparison with the experimental data (Table 1). Although the DFT results for the high-spin complexes using the pure density functional BP86 method somewhat overestimate the covalency of each complex, on average, by $\sim 6 \%$ for the ferric complexes (Table 1, columns 8 and 9), the DFT calculations nicely reflect the differential orbital covalencies (Table 1, column 10). For each of the complexes, the covalency of the $\sigma$ symmetry orbitals is higher than the $\pi$ orbitals, mirroring the experimental trend found from the L-edge DOC projection method and chemical intuition. For the high-spin ferric $O_{h}$ complexes, the calculated metal $t_{2 g}$ component is in the range of $83-85 \%$ and the metal $\mathrm{d}$ component in the $\mathrm{e}_{\mathrm{g}}$ symmetry orbitals was calculated to be 64-68\% (Figure 6B and D), versus the experimentally found values of $88-90 \%$ and $70-73 \%$, respectively. The low-spin ferric $\left[\mathrm{Fe}(\operatorname{tacn})_{2}\right]^{3+}$ complex was calculated to have $93 \% \mathrm{~d}$ character in the half-occupied $\mathrm{t}_{2 \mathrm{~g}}$ orbital and $62 \%$ in the unoccupied $\mathrm{e}_{\mathrm{g}}$ orbitals (Figure 6E), relative to $99 \%$ and $63 \%$ experimentally. Similar results are found for the ferric tetrahedral complex, $\left[\mathrm{FeCl}_{4}\right]^{-}$(Figure 6A), although inverted due to the tetrahedral ligand field. The $t_{2}$ and e symmetry orbitals were calculated to have $68 \%$ and $77 \%$ metal d orbital character, as compared to $76 \%$ and $89 \%$ found from the L-edge methodology. As can be seen from Table 1, the general trends in the experimental results are reproduced in the MO calculations and differences in the experimentally and theoretically calculated DOC are found to be $\leq 5 \%$ for the ferric complexes.

For the relatively less covalent ferrous complexes, again, the DFT results somewhat overestimate the covalency of each complex by $3 \%$ on average (Table 1 , columns 8 and 9) but nicely reflect the differential orbital covalencies (Table 1, column 10). For the ferrous tetrahedral complex $\left[\mathrm{FeCl}_{4}\right]^{2-}$ (Figure 6C), the $t_{2}$ and e symmetry orbitals were calculated to have $84 \%$ and $89 \%$ metal d orbital character, as compared to $87 \%$ and $93 \%$ determined from the L-edge methodology. The octahedral ferrous $\left[\mathrm{FeCl}_{6}\right]^{4-}$ complex was calculated to have $94 \%$ metal $d$ character in the half-occupied $\mathrm{t}_{2 \mathrm{~g}}$ orbitals and $83 \%$ in the half-occupied $\mathrm{e}_{\mathrm{g}}$ orbitals, while, from the iron L-edge DOC methodology, the $t_{2 g}$ and $e_{g}$ orbitals are found to have $96 \%$ and $88 \%$ metal d character, respectively. The low-spin $\left[\mathrm{Fe}(\operatorname{tacn})_{2}\right]^{2+}$ complex with the $\mathrm{t}_{2 \mathrm{~g}}$ orbitals completely filled and the $e_{g}$ orbitals unoccupied is calculated to be $71 \%$ metal and found from the data to be $72 \%$ metal d character. Generally good agreement, $\leq 3 \%$ for the ferrous complexes in this study, is found between the experimentally and theoretically determined DOC values. Most importantly, the experimental trends in the high- and low-spin, $O_{h}$ and $T_{d}$ complexes are reproduced by the calculations, indicating that these can provide further insight into the associated bonding differences.

\section{Discussion}

For the L-edge XAS spectrum of first-row transition metal complexes where the interaction between core and valence electrons is strong, the absorption spectrum is strongly affected by the $2 \mathrm{p}-3 \mathrm{~d}$ multiplet interactions. The spin-orbit coupling
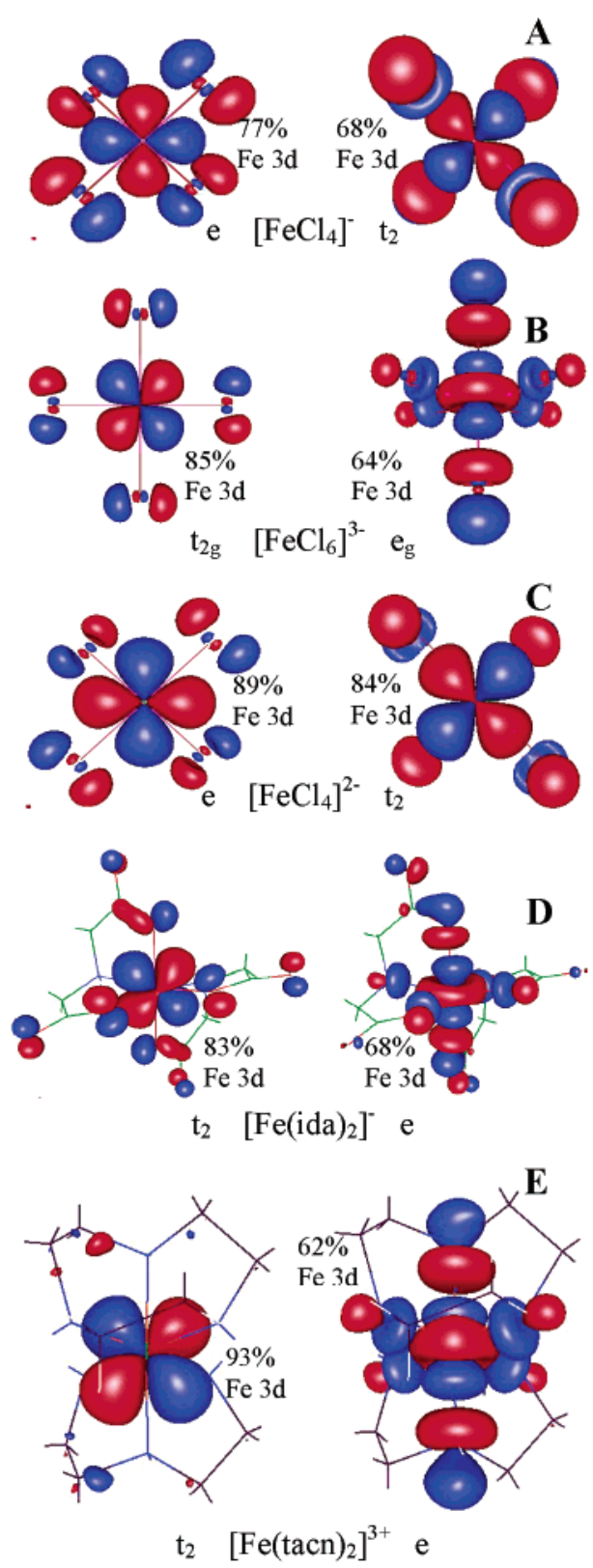

Figure 6. Contour plots of antibonding metal $\mathrm{d} \pi$ (left) and $\mathrm{d} \sigma$ (right) orbitals of (A) $\left[\mathrm{FeCl}_{4}\right]^{-}$, (B) $\left[\mathrm{FeCl}_{6}\right]^{3-}$, (C) $\left[\mathrm{FeCl}_{4}\right]^{2-}$, (D) $\left[\mathrm{Fe}(\mathrm{ida})_{2}\right]^{-}$, and (E) $\left[\mathrm{Fe}(\operatorname{tacn})_{2}\right]^{3+}$. The $\mathrm{t}_{2} \sigma$ orbitals of $\left[\mathrm{FeCl}_{4}\right]^{-}$and $\left[\mathrm{FeCl}_{4}\right]^{2-}$ have been rotated $90^{\circ}$ relative to the e $\pi$ orbitals for clarity.

of the metal $2 p$ core hole, the spin-orbit interactions of the $\mathrm{d}$-manifold, exchange interactions between the $2 \mathrm{p}$ and $3 \mathrm{~d}$ manifolds as well as within the $3 \mathrm{~d}$ manifold, and ligand field energy splitting all influence the experimental spectra. ${ }^{64}$ For iron model complexes with a number of half- or unoccupied orbitals in the valence d-manifold that result in a number of manyelectron excited state configurations, these multiplet effects distribute L-edge intensity across an energy range of approximately $20 \mathrm{eV}$.

The use of metal L-edge XAS intensity in determining covalency has been demonstrated in earlier studies, for both model complexes and protein systems. The covalency results derived from $\mathrm{Cu}$ L-edge studies have, for example, been used in conjunction with other spectroscopies to determine the metal $\mathrm{d}$ contribution to the ground state wave functions of certain blue 
copper ${ }^{17}$ and $\mathrm{Cu}_{\mathrm{A}}$ proteins ${ }^{18}$ and copper model complexes. ${ }^{19-21}$ For complexes with a $\mathrm{d}^{9}$ ground state electronic configuration, however, the $\mathrm{d}^{10}$ final state precludes any influence from multiplet interactions on the spectral shape; the L-edge transition consists of a single bound state transition for each of the $\mathrm{L}_{3^{-}}$ and $\mathrm{L}_{2}$-edges that can be integrated and used in an intensity/ covalency analysis. However, for iron model complexes with additional half- or unoccupied metal d orbitals, the multiplet effects obscure the precise energy of the ionization edge and complicate the intensity analysis of the spectra.

The metal L-edge intensity is proportional to the number of allowed one-electron transition pathways and consequently is dependent on the number of half- or unoccupied metal d orbitals. The experimental intensity must therefore be renormalized to reflect the intensity due to a single transition pathway. The dipole allowed metal $2 \mathrm{p} \rightarrow \Psi^{*}$ L-edge transitions are also proportional to the amount of metal d-character in the ground state wave function $\left(1-\alpha^{2}\right.$ in eq 1.1). As the amount of metal character in the ground state wave function decreases, the metal L-edge intensity also decreases. Thus, the total intensity of the iron L-edge is a direct probe of the average covalency of the half- and unoccupied iron d orbitals.

Using the multiplet program developed by Thole and coworkers, ${ }^{24}$ the metal L-edge spectrum may be simulated and the parameters used to describe the ground state wave function. The simulations include all multiplet and ligand field effects but include covalency effects only through the apparent modification of the multiplet parameters $\beta$ and $10 D q$. Covalency as metal-ligand overlap is modeled by further including CT shake-up states onto the multiplets. As shown in the ferric complexes studied above, certain L-edge spectral features cannot be simulated using an isotropic covalency. It was therefore essential to develop a methodology to include differential orbital covalency (DOC). Using the projection method (vide supra), the ground state wave function is projected onto a hypothetical excited state devoid of multiplet effects. Thus, the individual LMCT contributions to the orbitals of $t_{2}$ and e symmetry are obtained. Renormalizing these intensities to account for the number of $t_{2}$ and e holes determines the covalency of the individual orbital sets and therefore the differential orbital covalency of a metal active site. The DOC obtained in this way compares well to the results from other spectroscopies and DFTbased MO calculations.

The high-spin ferrous complexes are in general less covalent than the high-spin ferric complexes, as expected. The higher effective nuclear charge of the ferric complexes shifts the metal d-manifold to deeper binding energy and allows for a closer energetic match between half-occupied metal $d$ and occupied ligand $\mathrm{p}$ orbitals. The higher $\mathrm{Z}_{\mathrm{eff}}$ also results in a radial contraction of the metal d orbitals that differentially limits the overlap of the metal $d \pi$ orbitals with those of the ligand (Figure $6 \mathrm{~A}$ and $\mathrm{C}$ ). The result is a differential increase in covalency upon oxidation. The $\mathrm{d} \pi$ symmetry orbitals of the ferrous and ferric $T_{d}$ and $O_{h}$ chloride complexes have similar covalencies (Table 1). The $\sigma$ orbitals, however, become much more covalent upon oxidation, by $11 \%$ in the $T_{d}$ chloride and by $18 \%$ in the $O_{h}$ chloride. Thus, the increased average covalency of the ferric relative to the ferrous high-spin chloride complexes is due mainly to the increased ligand component in the $\mathrm{d} \sigma$ symmetry orbitals resulting from the increased $Z_{\text {eff }}$.
The high-spin ferrous tetrahedral and octahedral chloride complexes are quite ionic in nature (Table 1). The octahedral complex would be expected to be more covalent than the tetrahedral complex with two additional ligand donors, yet experimentally their covalencies are quite similar. The crystal structures of $\left[\mathrm{FeCl}_{4}\right]^{2-}$ and $\left[\mathrm{FeCl}_{6}\right]^{4-}$ reveal that the bond lengths of $\left[\mathrm{FeCl}_{4}\right]^{2-}(2.28 \AA)$ are $0.23 \AA$ shorter than those of $\left[\mathrm{FeCl}_{6}\right]^{4-}$ at $2.51 \AA .{ }^{30,67}$ The increased ligand-ligand repulsion results in longer bond lengths in the $O_{h}$ compound and reduces the overlap between the metal $d$ and ligand p orbitals, offsetting the greater number of metal-ligand interactions.

Interesting trends are observed in the high-spin ferric complexes studied. As with the case of the ferrous chloride complexes, the average covalencies of the $O_{h}$ and $T_{d}$ ferric chloride complexes are also quite similar. Analogous to the ferrous case, the bond lengths of the ferric $T_{d}$ complex $(2.18$ $\AA)$ are $\sim 0.2 \AA$ shorter than the $O_{h}$ complex $(2.39 \AA),{ }^{34,68}$ reflecting decreased ligand-ligand repulsion. This increases the metal-ligand overlap resulting in an increased covalency, despite the decrease in coordination number. The DOC of the $T_{d}$ ferric chloride (13\%) is significantly lower than that of the $O_{h}$ ferric chloride (25\%), however, due to the relatively stronger $\pi$ donor interaction in the $T_{d}$ complex at its shorter bond lengths (Figure 6A and B). The average covalencies of the remaining ferric octahedral high-spin complexes, $\left[\mathrm{Fe}(\mathrm{ox})_{3}\right]^{3-}, \mathrm{Fe}(\mathrm{acac})_{3}$, and $\left[\mathrm{Fe}(\mathrm{ida})_{2}\right]^{-}$, are similar to that of the $\mathrm{O}_{h}$ chloride, while the differential orbital covalencies are significantly less than that of the $O_{h}$ chloride due to both reduced $\sigma$ and increased $\pi$ covalencies in these complexes.

In the low-spin iron complexes studied here with predominantly $\sigma$ donating ligands, the relatively ionic $\mathrm{t}_{2 \mathrm{~g}}$ orbitals are mostly or completely filled, and the $\mathrm{e}_{\mathrm{g}}$ orbitals of much higher covalency are unoccupied. Thus the average covalency is found to be much greater for the low-spin iron complexes. In general, the higher $Z_{\text {eff }}$ of the ferric complex would tend to increase the relative contribution of the ligand to the ground state and therefore increase the covalency of the ferric complex over that of the ferrous complex. Experimentally, however, the $[\mathrm{Fe}$ $\left.(\operatorname{tacn})_{2}\right]^{2 / 3+}$ complexes are found to have comparable average covalencies. For a low-spin $\mathrm{d}^{6} O_{h}$ complex, the average covalency derived from the integrated L-edge intensity is an average of the four highly covalent unoccupied $e_{g}$ orbitals. The covalency for the ferric complex, however, is the average of the four $\sigma$ orbitals as well as the one $\pi$ orbital. The impact of the additional half-occupied $t_{2 g}$ orbital in the ferric complex is easily observed from the DOC analysis. The increased $Z_{\text {eff }}$ in the oxidized complex results in $e_{g}$ orbitals which are in fact more covalent with $63 \%$ metal d character as compared to only $72 \%$ metal d character in the reduced complex. Since the tacn ligand has no $\pi$ orbitals available for mixing with the metal $d$ orbitals, the $t_{2 g}$ orbitals are nearly $100 \%$ ionic. Thus, the DOC in the ferric low-spin $\left[\mathrm{Fe}(\operatorname{tacn})_{2}\right]^{3+}$ is much larger than what is found for the high-spin complexes. As the DOC analysis reflects the covalencies of unoccupied molecular orbitals, a precise determination of DOC is not possible for a low-spin ferric complex with $\mathrm{a} \mathrm{t}_{2 \mathrm{~g}}{ }^{6}$ ground state. Based on comparisons with the high-spin ferrous systems, the $\pi$ covalency of the low-spin $\left[\mathrm{Fe}(\mathrm{tacn})_{2}\right]^{3+}$ complex can be estimated at $0-4 \%$ ligand

(67) Figgis, B. N.; Sobolev, A. N.; Kucharski, E. S.; Broughtaon, V. Acto Crystallogr. 2000, C56, E228-E229.

(68) Cotton, F. A.; Murillo, C. A. Inorg. Chem. 1975, 14, 2467-2469. 
character. Thus, the DOC of $\left[\mathrm{Fe}(\operatorname{tacn})_{2}\right]^{2+}$ can be estimated at $24 \%-28 \%$, far higher than in the high-spin ferrous systems.

The average metal d character determined from DFT calculations is $5-10 \%$ lower than that found experimentally, consistent with the fact that a pure DFT approach is generally overly covalent. However, the DFT calculations nicely follow the trends seen in the L-edge methodology developed herein. The calculations reproduce not only the low covalency of the ferrous site (8-10\% experimentally) but also the minor DOC, found to be $\leq 3 \%$. Furthermore, the $O_{h}$ chloride is found from both experiment and DFT to be slightly less covalent than the $T_{d}$ complex. The change in covalency observed experimentally between the ferric $T_{d}$ and $O_{h}$ chlorides (4\%) is also observed in the calculational results $(5 \%)$. The remaining high-spin ferric $O_{h}$ complexes are all observed to have a lower DOC than that of the $O_{h}$ chloride complex. The DFT results provide insights into this trend. First, the $\pi$ covalency is increased due to the presence of low-lying $\pi$ donor orbitals in the acac, ida, and ox ligands. Furthermore, the $\sigma$ donor interaction is decreased. While this is clearly complicated by the differences in $2 p$ versus $3 p$ radial distribution functions of the $\mathrm{O}-\mathrm{Fe}^{\mathrm{III}}$ at $\sim 2.00 \AA$ versus $\mathrm{Cl}-$ $\mathrm{Fe}^{\mathrm{III}}$ at $2.39 \AA$, an important contribution can be seen in Figure $6 \mathrm{D}$ which shows that the oxygen $\sigma$ valence donor orbital is partly delocalized on the carbon framework of the chelate which reduces the donor interaction of the $\sigma$ orbital with the iron.

The low-spin $\sigma$-donor complexes studied here also demonstrate similar trends between DFT calculations and experimental data. While the low-spin $\left.\left[\mathrm{Fe}(\operatorname{tacn})_{2}\right)\right]^{2+}$ complex has no unoccupied $\mathrm{t}_{2}$ orbitals, the e symmetry orbitals are found to have $72 \%$ and $71 \%$ metal d character from experiment and calculations, respectively. The ferric low-spin complex, with a single half-occupied $t_{2}$ orbital and four spin-unrestricted unoccupied e orbitals, was found from the L-edge methodology to be $2 \%$ more covalent $(70 \%)$ than the ferrous complex. DFT results indicate a similar trend, finding the average covalency to be $68 \%$ metal d character, 3\% more covalent than its ferrous counterpart. More striking is the fact that trends in the DOC determined from the experiment and DFT also are quite similar for the $\left.\left[\mathrm{Fe}(\operatorname{tacn})_{2}\right)\right]^{3+}$ complex. Paralleling the experimental results, the DFT calculations reproduce the total average covalency of the $\left[\mathrm{Fe}(\operatorname{tacn})_{2}\right]^{2 / 3+}$ complexes and show that the half-occupied $t_{2} \pi$ orbital is relatively ionic since the tacn ligand has no $\pi$ orbitals available for bonding (Figure $6 \mathrm{E}$ ). The results further show that the $\sigma$ orbitals are indeed more covalent in the ferric complex (62\% metal d character) as a result of the higher effective nuclear charge.

\section{Conclusions}

In this study, the integrated intensity approach previously developed for systems with a single hole in the $\psi^{*}$ SOMO orbital of $\mathrm{Cu}$ proteins and model complexes has been expanded to iron model complexes. The intensity of the iron L-edge data is renormalized to account for the one-electron transition pathways available into the additional half- or unoccupied $d$ orbitals in the $3 \mathrm{~d}^{5}$ or $3 \mathrm{~d}^{6}$ site. This enables the quantitative determination of the overall covalency of the complex, which is corroborated by additional spectroscopies and molecular orbital theory. Using this methodology, the total covalencies of a series of ferric and ferrous, high- and low-spin model complexes were determined. Ferrous high-spin complexes were found to be mostly ionic in nature, while ferric high-spin and $\sigma$-donating low-spin complexes were found to be much more covalent. The data were simulated using the charge transfer multiplet model with e and $t_{2}$ symmetry shake-ups, and the simulations were projected into a hypothetical state, where multiplet effects are eliminated, permitting determination of the differential orbital covalency of the individual symmetry related $\mathrm{d}$ orbitals of the metal. While the DOC determined for ferrous high-spin complexes is quite low in accordance with their low average covalency, significant differences in the $t_{2 g}$ and $e_{g}$ orbital covalency are found for ferric high- and low-spin octahedral complexes, where the high covalency of the $\mathrm{e}_{\mathrm{g}}$ orbitals of $\left[\mathrm{Fe}(\operatorname{tacn})_{2}\right]^{3+}$ results in a very high DOC of $36 \%$.

The sensitivity of the DOC to high- and low-spin ferric sites lends itself well to the study of other complexes of significance in bioinorganic chemistry. The average covalency determined from integrating the L-edge intensity, coupled with the DOC methodology developed herein, can provide a quantitative measurement of the $\pi$-bonding interaction of the $\mathrm{Fe}-\mathrm{O}$ bond in siderophores which has been considered $^{69}$ to make a significant contribution to the high stability $\left(K_{\mathrm{f}} \approx 10^{25-50}\right)$ in these complexes. Alternatively, the DOC from the metal L-edge analysis can provide a detailed, quantitative description of the difference of in-plane versus out-of-plane $\pi$ bonding in lowspin ferrihemes, which from EPR studies can give rise to two different ground states $\left(\mathrm{d}_{x z, y z}\right.$ or $\left.\mathrm{d}_{x y}\right)$. Finally, DOC analysis can further elucidate the nature of the $\pi$ back-bonding to the lowlying ligand $\pi^{*}$ orbitals commonly associated with the iron cyanides which should produce unique features in the L-edge spectrum. Finally, DOC analysis can be a crucial aid in studying the short $(\sim 1.65 \AA)$ iron-oxo bond in ferric and ferryl heme relative to non-heme iron sites where intense CT shake-up features associated with the oxo $\sigma$ and $\pi$ interactions can greatly perturb their electronic structures.

Acknowledgment. Professor Roland Meier is thanked for providing the $\left[\mathrm{Fe}(\mathrm{ida})_{2}\right]^{-}$compound used in this study. This work was supported by grants from the National Institutes of Health: GM40392 to E.I.S. and RR-01209 to K.O.H. SSRL operations and research are funded by the U.S. Department of Energy (Office of Basic Energy Science). The Structural Molecular Biology program at SSRL is funded by the National Institutes of Health, National Center for Research Resources, Biomedical Technology Program, and the Department of Energy, Office of Biological and Environmental Research.

Supporting Information Available: Data and DOC simulations of $\left[\mathrm{FeCl}_{4}\right]^{-},\left[\mathrm{FeCl}_{4}\right]^{2-}, \mathrm{Fe}(\mathrm{acac})_{3},\left[\mathrm{Fe}(\mathrm{ox})_{3}\right]^{3-}$, and $\left[\mathrm{Fe}(\mathrm{ida})_{2}\right]^{-}$, as well as ligand $\mathrm{K}$-edge data and covalency analysis for $\left[\mathrm{FeCl}_{6}\right]^{3-}$. This material is available free of charge via the Internet at http://pubs.acs.org.

\section{JA034634S}

(69) Karshipin, T. B.; Gebhard, M. S.; Solomon, E. I.; Raymond, K. N. J. Am. Chem. Soc. 1991, 113, 2977-2984 